\title{
Invariant Manifolds for Abstract Functional Differential Equations and Related Volterra Difference Equations in a Banach Space
}

\author{
By \\ Satoru MuraKami* and Yutaka NagabuchI \\ (Okayama University of Science, Japan)
}

\begin{abstract}
For abstract functional differential equations (FDE) and Volterra difference equations (VDE) in a Banach space, the local existence and smoothness of invariant manifolds, such as stable/unstable manifolds, center-stable/center-unstable manifolds and center manifolds, are established by means of the variation of constants formula in the phase space in [18] and [12]. Also, it is shown that in a neighborhood of the zero solution the behavior of solutions of FDE (resp. VDE) is described, in some sense, by a certain ordinary differential equation (resp. first order difference equation) in a finite dimensional space. As a corollaly, the principle of linearized stability is derived.

Key Words and Phrases. Abstract functional differential equations, Volterra difference equations, Invariant manifolds, Principle of linearized stability.

2000 Mathematics Subject Classification Numbers. Primary 39A10, 39A11; Secondary $34 \mathrm{~K} 20,35 \mathrm{~B} 35$.
\end{abstract}

\section{Introduction}

In this paper we are concerned with the abstract functional differential equation in a Banach space $X$

$$
\dot{x}(t)=A x(t)+L\left(x_{t}\right)+f\left(x_{t}\right),
$$

where $A$ is the infinitesimal generator of a strong continuous compact semigroup $\{T(t)\}_{t \geq 0}$ on a Banach space $X, x_{t}$ is an element of $\mathscr{B}$ defined by $x_{t}(\theta):=$ $x(t+\theta)$ for $\theta \in(-\infty, 0], L: \mathscr{B} \rightarrow X$ is a bounded linear operator, $f \in C^{k}(\mathscr{B} ; X)$ with $f(0)=D f(0)=0$, and $\mathscr{B}$ is the phase space for Eq. (1) satisfying some fundamental axioms introduced in section 2. We also consider the Volterra difference equation, a discrete analogue of (1),

$$
x(n+1)=\sum_{j=-\infty}^{n} Q(n-j) x(j)+g\left(x_{n}\right),
$$

\footnotetext{
* Partly supported by the Grant-in-Aid for Scientific Research (C), No. 16540177, The Ministry of Education, Science, Sports and Culture, Japan.
} 
where $\{Q(n)\}_{n \geq 0}$ is a sequence of compact linear operators on $X$ satisfying the condition $\sum_{n=0}^{\infty}\|Q(n)\|_{\mathscr{L}(X)} e^{\gamma n}<\infty$ for some $\gamma>0, g$ is a $C^{k}$-mapping from $\mathscr{B}^{\gamma}$, the phase space for Eq. (2) (see section 2), to $X$ with $g(0)=D g(0)=0$, and $x_{n}$ is an element in $\mathscr{B}^{\gamma}$ given by $x_{n}(\theta):=x(n+\theta)$ for $\theta \in\{0,-1,-2, \ldots\}$.

In the study of dynamics near an equilibrium of nonlinear differential equations and difference equations, invariant manifolds, such as stable manifolds and unstable manifolds, play important roles, and among them center-unstable manifolds, together with center manifolds, are crucial for determination of fine structures of the dynamics when the equilibrium is nonhyperbolic $[3,6,7,13$, 15, 32]. Two types of approaches are known to establish exsistence theorems of such invariant manifolds: the Liapunov-Perron method constructs the manifold as the set of points, each of which corresponds to a solution of a suitable integral (resp. Volterra difference) equation closely related to the differential (resp. difference) equation under consideration (see, e.g., $[3,6,7,13,15]$ ); while the Hadamard method formulates the existence of the manifold as a fixed point problem of so called the graph transform (see [20, 31]).

On the existence of some invariant manifolds for Eq. (1), there are many research papers; we refer the reader to $[2,6,15,16,26,32]$ and the references therein. In a preceding paper [29] the first author and Nguyen V. Minh employed the variation of constants formula in the phase space $\mathscr{B}$, established in [18], and proved the existence and Lipschitz smoothness (in fact, $C^{1}$ smoothness) of stable, unstable and center-unstable manifolds for the zero solution of Eq. (1). Also by using the Liapunov-Perron method, Krisztin [21] studied some invariant manifolds for time- $t$ map, a discretization of a semiflow, together with their invariance under the semiflow, and succeeded in showing the existence of smooth local center-stable, center-unstable and center manifolds for Eq. (1), developing the method employed in the book [22] where some $C^{1}$-smooth invariant manifolds are studied for some scalar functional differential equations. Moreover, the recent work of Nguyen V. Minh and Wu [27] has obtained smooth local center-unstable and center manifolds for Eq. (1) based on the Hadamard method.

On the other hand, Volterra difference equations have also been studied widely by many reseachers (see $[1,4,5,7,8,9,25]$ and the references therein). The authors $[10,11,12]$ proved some fundamental results on the asymptotic stability of the homogeneous equation of (2) in connection with the invertibility of the characteristc operator in the case where $X$ is of infinite dimension, and generalized some of the results in [7, 8]. For the existence of invariant manifolds for Eq. (2), we mention to a recent paper by Matsunaga and Murakami [24], which showed the existence of stable, unstable and centerunstable manifolds, together with their Lipschitz smoothness, when $X$ is of finite dimension. 
The main purpose of the present paper is to improve the results in [29], that is, to establish the existence of smooth local stable, unstable, center-stable, center-unstable and center manifolds for Eq. (1), by means of variation of constants formula in the phase space, mentioned above (see also [28]), together with Diekmann's technique [6]. We note that our approach based on variation of constants formula in the phase space has the advantage that it readily enables us to describe explicitly the dynamics on center-unstable manifolds (or center manifolds) in terms of certain ordinary differential equations in Euclidean spaces.

We shall, in addition, discuss the corresponding problem for Volterra difference equation (2) and, by a similar approach, establish some existence theorems of smooth local invariant manifolds for the zero solution. In this point we should mention to the book [22] again, where the existence and $C^{1}$-smoothness of several invariant manifolds for discerte dynamical systems generated by maps, as well as their applications to some scalar functional differential equations, are discussed in a framework of general Banach spaces. It is possible to apply their argument to existence problem of $C^{1}$-smooth invariant manifolds for Eq. (2), and besides, their method also seems to be available for the verification of further smoothness. We note again that our method has the same advantage for Eq. (2) as for Eq. (1); see Theorem 12.

This paper is outlined as follows: In the next section we give some preparatory results, for both Eq. (1) and (2), on the phase space, variation of constants formula in the phase space, decomposition of the phase space and so on. In section 3 we prove the existence of $C^{k}$-smooth local stable and unstable manifolds for Eq. (1), and establish in section 4 that of $C^{k}$-smooth centerunstable manifolds. We give in section 5 the corresponding results on center and center-stable manifolds with sketch of their proofs. Section 6 shows analogous theorems for Volterra difference equation (2) and, as their applications, we state in the last section the principle of linearized stability for Volterra difference equations.

\section{Notations and some preparatory results}

Let $\boldsymbol{Z}, \boldsymbol{Z}^{+}, \boldsymbol{Z}^{-}, \boldsymbol{R}$ and $\boldsymbol{C}$ be the set of integers, nonnegative integers, nonpositive integers, real numbers and complex numbers, respectively. For an interval $J \subset \boldsymbol{R}$ and a Banach space $U$ we denote by $C(J ; U)$ the space of $U$-valued continuous functions on $J$, and by $B C(J ; X)$ its subspace of bounded continuous functions on $J$. Also, for Banach spaces $U, W$ and positive integer $p$, let us denote by $\mathscr{L}^{p}(U ; W)$ the space of bounded $p$-linear mappings from $U$ to $W$ with norm

$$
\|Q\|_{\mathscr{L}^{p}(U ; W)}:=\sup \left\{\left\|Q\left(u_{1}, \ldots, u_{p}\right)\right\|_{W}:\left\|u_{j}\right\|_{U} \leq 1, u_{j} \in U, j=1, \ldots, p\right\}
$$


for $Q \in \mathscr{L}^{p}(U ; W)$. In particular, we use the notation $\mathscr{L}(U ; W)$, the space of bounded linear operators, rather than $\mathscr{L}^{1}(U ; W)$; and simply write $\mathscr{L}(U)$ in place of $\mathscr{L}(U ; U)$.

\subsection{Phase spaces}

We shall first introduce the phase spaces for Eq. (1). Let us denote by $\|\cdot\|_{X}$ the norm of $X$. For any function $x:(-\infty, a) \rightarrow X$ and $t<a$, we define $x_{t}: \boldsymbol{R}^{-}:=(-\infty, 0] \rightarrow X$ by $x_{t}(\theta)=x(t+\theta)$ for $\theta \in \boldsymbol{R}^{-}$. A Banach space $\mathscr{B}$ with norm $\|\cdot\|_{\mathscr{B}}$ consisting of functions from $\boldsymbol{R}^{-}$to $X$ is called a fading memory space if it satisfies the following properties:

(A1) There exist a positive constant $N$ and locally bounded functions $K(\cdot)$ and $M(\cdot)$ on $\boldsymbol{R}^{+}:=[0, \infty)$ with the property that if $x:(-\infty, a) \rightarrow X$ is continuous on $[\sigma, a)$ with $x_{\sigma} \in \mathscr{B}$ for some $\sigma<a$, then for all $t \in[\sigma, a)$,

(i) $x_{t} \in \mathscr{B}$,

(ii) $x_{t}$ is continuous in $t$ (w.r.t. $\|\cdot\|_{\mathscr{B}}$ ),

(iii) $N\|x(t)\|_{X} \leq\left\|x_{t}\right\|_{\mathscr{B}} \leq K(t-\sigma) \sup _{\sigma \leq s \leq t}\|x(s)\|_{X}+M(t-\sigma)\left\|x_{\sigma}\right\|_{\mathscr{B}}$

(A2) If $\left\{\phi^{k}\right\}, \phi^{k} \in \mathscr{B}$, converges to $\phi$ uniformly on any compact set in $\boldsymbol{R}^{-}$ and if $\left\{\phi^{k}\right\}$ is a Cauchy sequence in $\mathscr{B}$, then $\phi \in \mathscr{B}$ and $\phi^{k} \rightarrow \phi$ in $\mathscr{B}$.

A fading memory space $\mathscr{B}$ is called a uniform fading memory space, if it satisfies (A1) and (A2) with $K(\cdot) \equiv K$ (a constant) and $M(\beta) \rightarrow 0$ as $\beta \rightarrow \infty$ in (A1). The Banach space

$$
C_{\lambda}=C_{\lambda}(X)=\left\{\phi \in C\left(\boldsymbol{R}^{-} ; X\right): \lim _{\theta \rightarrow-\infty}\|\phi(\theta)\|_{X} e^{\lambda \theta}=0\right\}, \quad \lambda>0
$$

endowed with norm $\|\phi\|_{C_{\lambda}}=\sup _{\theta \leq 0}\|\phi(\theta)\|_{X} e^{\lambda \theta}$ is one of typical examples of uniformly fading memory space.

It is known [14, Lemma 3.2] that if $\mathscr{B}$ is a uniform fading memory space, then $\mathrm{BC}:=B C\left(\boldsymbol{R}^{-} ; X\right) \subset \mathscr{B}$ and the inclusion map from $\mathrm{BC}$ into $\mathscr{B}$ is continuous. For other properties of fading memory spaces and uniform fading memory spaces, the reader may consult for the book [17].

We next consider the Volterra difference equation (2). Set

$$
\mathscr{B}^{\gamma}:=\left\{\phi: \boldsymbol{Z}^{-} \rightarrow X: \sup _{\theta \in \boldsymbol{Z}^{-}}\|\phi(\theta)\|_{X} e^{\gamma \theta}<\infty\right\}
$$

for $\gamma>0$. Then, $\mathscr{B}^{\gamma}$ is a Banach space with norm $\|\phi\|_{\mathscr{B}^{\gamma}}=\sup _{\theta \in \boldsymbol{Z}^{-}}\|\phi(\theta)\|_{X} e^{\gamma \theta}$, which plays the role of phase space for Eq. (2) under our assumption that $\sum_{n=0}^{\infty}\|Q(n)\|_{\mathscr{L}(X)} e^{\gamma n}<\infty$ (see [12], [30]). Throughout the paper $\mathscr{B}^{\gamma}$ will be employed as the phase space, whenever the behavior of solutions of Eq. (2) is discussed. 


\subsection{A Variation of constant formula and decomposition of the phase space for FDE}

Consider the nonhomogeneous functional differential equation

$$
\dot{x}(t)=A x(t)+L\left(x_{t}\right)+h(t)
$$

where $h \in C(\boldsymbol{R} ; X)$. For any $(\sigma, \phi) \in \boldsymbol{R} \times \mathscr{B}$, there exists a unique function $x: \boldsymbol{R} \rightarrow X$ such that $x_{\sigma}=\phi, x$ is continuous on $[\sigma, \infty)$ and the following relation holds:

$$
x(t)=T(t-\sigma) \phi(0)+\int_{\sigma}^{t} T(t-s)\left\{L\left(x_{s}\right)+h(s)\right\} d s, \quad t \geq \sigma .
$$

The function $x$ is called a (mild) solution of (3) through $(\sigma, \phi)$ on $[\sigma, \infty)$, and denoted by $u(\cdot, \sigma, \phi ; h)$. When $J$ is an interval in $\boldsymbol{R}$, a function $\xi$ is called a solution of Eq. (3) on $J$, if $\xi_{t} \in \mathscr{B}$ is defined for all $t \in J$ and if it satisfies $x\left(t, \sigma, \xi_{\sigma} ; h\right)=\xi(t)$ for all $t$ and $\sigma$ in $J$ with $t \geq \sigma$.

For $t \geq 0$ we define an operator $V(t)$ on $\mathscr{B}$ by

$$
V(t) \phi:=x_{t}(0, \phi ; 0), \quad \phi \in \mathscr{B} .
$$

We can readily see that $\{V(t)\}_{t \geq 0}$ is a strongly continuous semigroup of bounded linear operators on $\mathscr{B}$, called the solution semigroup of (3).

Given a positive integer $n$, let us define a function $\Gamma^{n}$ by

$$
\Gamma^{n}(\theta):= \begin{cases}(n \theta+1) I_{X}, & -1 / n \leq \theta \leq 0 \\ 0, & \theta<-1 / n\end{cases}
$$

where $I_{X}$ is the identity mapping on $X$. It follows from (A1) that if $x \in X$, then $\Gamma^{n} x \in \mathscr{B}$ with $\left\|\Gamma^{n} x\right\|_{\mathscr{B}} \leq K(1)\|x\|_{X}$.

The following theorem gives a representation formula for solutions of (3) in the phase space $\mathscr{B}$, called the variation of constants formula (abbreviated as $\mathrm{VCF}$ ) in the phase space, which is an essential tool in the present paper.

Theorem 1 ([28]). The segment $x_{t}(\sigma, \phi ; h)$ of solution $x(\cdot, \sigma, \phi ; h)$ of (3) satisfies the following relation in $\mathscr{B}$ :

$$
x_{t}(\sigma, \phi ; h)=V(t-\sigma) \phi+\lim _{n \rightarrow \infty} \int_{\sigma}^{t} V(t-s) \Gamma^{n} h(s) d s, \quad t \geq \sigma .
$$

Here and subsequently we will assume that $\mathscr{B}$ is a uniform fading memory space, $A$ is the generator of a compact semigroup $\{T(t)\}_{t \geq 0}$ and $f \in C^{k}(\mathscr{B} ; X)$ with $f(0)=D f(0)=0$, where $k$ is a positive integer.

The zero solution of the linearized equation of (1)

$$
\dot{x}(t)=A x(t)+L\left(x_{t}\right)
$$


is said to be hyperbolic, if the null space of its characteristic operator $A-\lambda I_{X}-$ $L\left(e^{\lambda} I_{X}\right)$ is trivial for any $\lambda \in C$ with $\Re \lambda=0$. By virtue of the compactness of $T(t)$ one may say that the zero solution is hyperbolic if the characteristic operator is invertible in $\mathscr{L}(X)$ for all such $\lambda \in \boldsymbol{C}$.

Now consider the set of $C$

$$
\Sigma=\left\{\lambda \in C: \Re \lambda \geq 0 \text { and } A-\lambda I_{X}-L\left(e^{\lambda} I_{X}\right) \text { has a nontrivial null space }\right\} .
$$

Then, as discussed in $[18$, Section 5], to the set $\Sigma$ there corresponds a decomposition of the phase space

$$
\mathscr{B}=E^{s} \oplus E^{c} \oplus E^{u}
$$

where $E^{s}, E^{c}$ and $E^{u}$ are closed subspace of $\mathscr{B}$ which are invariant under the solution semigroup $\{V(t)\}_{t \geq 0}$; in particular $E^{c}$ and $E^{u}$ are finite dimensional subspaces corresponding to $\Sigma^{c}:=\Sigma \cap\{\lambda: \Re \lambda=0\}$ and $\Sigma^{u}:=\Sigma \cap\{\lambda: \Re \lambda>0\}$, respectively. Moreover, put $V^{u}(t)=\left.V(t)\right|_{E^{u}}, \quad V^{c}(t)=\left.V(t)\right|_{E^{c}}$ and $V^{s}(t)=$ $\left.V(t)\right|_{E^{s}}$. Then the following theorem holds:

Theorem 2 ([18]). Let $\{V(t)\}_{t \geq 0}$ be the solution semigroup of Eq. (4). Then there is a decomposition of the phase space

$$
\mathscr{B}=E^{s} \oplus E^{c} \oplus E^{u}
$$

with the following properties:

(i) $\operatorname{dim}\left(E^{u} \oplus E^{c}\right)<\infty$,

(ii) $V(t) E^{u} \subset E^{u}, V(t) E^{c} \subset E^{c}$, and $V(t) E^{s} \subset E^{s}$,

(iii) $V^{s}(t)$ has the semigroup property in $\boldsymbol{R}^{+}$, while $V^{u}(t)$ and $V^{c}(t)$ have in $\boldsymbol{R}$,

(iv) there exist positive constants $\alpha, \varepsilon$ with $\alpha>\varepsilon$ and a constant $C \geq 1$ such that

$$
\begin{aligned}
\left\|V^{s}(t)\right\|_{\mathscr{L}(\mathscr{B})} \leq C e^{-\alpha t}, & t \in \boldsymbol{R}^{+}, \\
\left\|V^{u}(t)\right\|_{\mathscr{L}(\mathscr{B})} \leq C e^{\alpha t}, & t \in \boldsymbol{R}^{-}, \\
\left\|V^{c}(t)\right\|_{\mathscr{L}(\mathscr{B})} \leq C e^{\varepsilon|t|}, & t \in \boldsymbol{R} .
\end{aligned}
$$

In (iv) we note that $C$ is a constant depending only on $\alpha$ and $\varepsilon$, and that the value of $\varepsilon>0$ can be taken arbitrarily small. Also, throughout the paper we use the notations $E^{c u}=E^{c} \oplus E^{u}, E^{c s}=E^{c} \oplus E^{s}$ etc; and denote by $\Pi^{s}$ the projection from $\mathscr{B}$ onto $E^{s}$ along $E^{c u}$, and similarly for $\Pi^{u}, \Pi^{c u}$ etc. Moreover, we set

$$
C_{1}:=\left\|\Pi^{s}\right\|_{\mathscr{L}(\mathscr{B})}+\left\|\Pi^{c}\right\|_{\mathscr{L}(\mathscr{B})}+\left\|\Pi^{u}\right\|_{\mathscr{L}(\mathscr{B})} .
$$




\subsection{VCF and decomposition of the phase space for VDE}

Consider the nonhomogeneous Volterra difference equation

$$
x(n+1)=\sum_{j=-\infty}^{n} Q(n-j) x(j)+h(n)
$$

where $h$ is an $X$-valued function defined on $\boldsymbol{Z}$. For any $(\sigma, \phi) \in \boldsymbol{Z} \times \mathscr{B}^{\gamma}$, the solution $x(n)$ of Eq. (5) satisfying the initial condition $x_{\sigma}=\phi$ is given by

$$
\begin{aligned}
x(n)= & R(n-\sigma) \phi(0)+\sum_{j=0}^{n-1} R(n-j-1) h(j) \\
& +\sum_{j=\sigma}^{n-1} R(n-j-1)\left(\sum_{k=-\infty}^{-1} Q(j-\sigma-k) \phi(k)\right)
\end{aligned}
$$

for $n \geq \sigma$, where $\{R(n)\}_{n \in Z^{+}}$is the fundamental solution of (5), that is, the sequence in $\mathscr{L}(X)$ defined by

$$
R(n+1)=\sum_{j=0}^{n} Q(n-j) R(j), \quad R(0)=I_{X}
$$

(see [10] and [12]). (We usually employ the convention $\sum_{\sigma}^{\sigma-1}=0$ for any $\sigma \in \boldsymbol{Z}$.) The function $x$ above is called the solution of (5) through $(\sigma, \phi)$ on $[\sigma, \infty) \cap \boldsymbol{Z}$, and denoted by $x(\cdot, \sigma, \phi ; h)$. When $J$ is an interval in $\boldsymbol{Z}$, a function $\xi$ is called a solution of Eq. (5) on $J$, if $\xi_{n} \in \mathscr{B}^{\gamma}$ is defined for all $n \in J$ and if it satisfies $x\left(n, \sigma, \xi_{\sigma} ; h\right)=\xi(n)$ for all $n$ and $\sigma$ in $J$ with $n \geq \sigma$.

For $n \in \boldsymbol{Z}^{+}$we define an operator $V(n)$ on $\mathscr{B}^{\gamma}$ by

$$
V(n) \phi:=x_{n}(0, \phi ; 0), \quad \phi \in \mathscr{B}^{\gamma} .
$$

We can readily see that $\{V(n)\}_{n \in Z^{+}}$is a semigroup of bounded linear operators on $\mathscr{B}^{\gamma}$, called the solution semigroup (or solution operator) of (5).

Let us define an operator $\mathscr{E}: X \rightarrow \mathscr{B}^{\gamma}$ by

$$
[\mathscr{E} x](\theta)= \begin{cases}x, & \theta=0, \\ 0, & \theta=-1,-2,-3, \ldots\end{cases}
$$

for any $x \in X$. Clearly, $\mathscr{E}$ is an isometry from $X$ into $\mathscr{B}^{\gamma}: \quad\|\mathscr{E} x\|_{\mathscr{B}}{ }^{\gamma}=\|x\|_{X}$ for $x \in X$. Corresponding to Theorem $1, \mathrm{VCF}$ in the phase space for Volterra difference equations is represented in the following form.

Theorem 3 ([12]). For any $\sigma \in \boldsymbol{Z}$ and $\phi \in \mathscr{B}^{\gamma}$ we have

$$
x_{n}(\sigma, \phi ; h)=V(n-\sigma) \phi+\sum_{k=\sigma}^{n-1} V(n-k-1) \mathscr{E} h(k), \quad n \geq \sigma .
$$


The zero solution of the linearized equation of (2)

$$
x(n+1)=\sum_{j=-\infty}^{n} Q(n-j) x(j)
$$

is said to be hyperbolic if for every $z \in C$ with $|z|=1$, the null space of its characteritic operator $z I_{X}-\sum_{n=0}^{\infty} Q(n) z^{-n}$ is trivial; this is equivalent to the invertibility of $z I_{X}-\sum_{n=0}^{\infty} Q(n) z^{-n}$ in $\mathscr{L}(X)$ since $Q(n), n \in \boldsymbol{Z}^{+}$, are compact.

Consider the set

$$
\Sigma=\left\{z \in C:|z| \geq 1 \text { and } z I_{X}-\sum_{n=0}^{\infty} Q(n) z^{-n} \text { has a no trivial null space }\right\},
$$

and its subsets $\Sigma^{c}:=\Sigma \cap\{z:|z|=1\}$ and $\Sigma^{u}:=\Sigma \cap\{z:|z|>1\}$. In [30] the decomposition result of the phase space, an analogue of Theorem 2, has been established for Eq. (6), which can be stated as follows:

Theorem 4 ([30]). Let $V(n)$ be the solution semigroup of Eq. (6). Then, to the set $\Sigma$ there corresponds a decomposition of the phase space

$$
\mathscr{B}^{\gamma}=E^{s} \oplus E^{c} \oplus E^{u}
$$

with the following properties:

(i) $\operatorname{dim}\left(E^{u} \oplus E^{c}\right)<\infty$,

(ii) $V(n) E^{u} \subset E^{u}, V(n) E^{c} \subset E^{c}$, and $V(n) E^{s} \subset E^{s}$,

(iii) $V^{s}(n)$ has the semigroup property in $\boldsymbol{Z}^{+}$, while $V^{u}(n)$ and $V^{c}(n)$ have in $\boldsymbol{Z}$,

(iv) there exist positive constants $\alpha, \varepsilon$ with $\alpha>\varepsilon$ and a constant $C \geq 1$ such that

$$
\begin{aligned}
\left\|V^{s}(n)\right\|_{\mathscr{L}(\mathscr{B})} \leq C e^{-\alpha n}, & n \in \boldsymbol{Z}^{+}, \\
\left\|V^{u}(n)\right\|_{\mathscr{L}(\mathscr{B})} \leq C e^{\alpha n}, & n \in \boldsymbol{Z}^{-}, \\
\left\|V^{c}(n)\right\|_{\mathscr{L}(\mathscr{B})} \leq C e^{\varepsilon|n|}, & n \in \boldsymbol{Z} .
\end{aligned}
$$

The notations $V^{s}, V^{c}$ and $V^{u}$ in the theorem are the same as those in Theoerm 2.

\subsection{Some Banach spaces of continuous functions}

We introduce Banach spaces which shall be used in the subsequent sections. Let $J$ denote one of the intervals $\boldsymbol{R}^{+}$and $\boldsymbol{R}^{-}$. For Banach space $U$ with norm $\|\cdot\|_{U}$ and $\lambda \in \boldsymbol{R}^{+}$, let us define 


$$
B C^{\lambda}(J ; U):=\left\{y \in C(J ; U): \sup _{t \in J}\|y(t)\|_{U} e^{\lambda t}<\infty\right\} .
$$

Then $B C^{\lambda}(J ; U)$ is a Banach space equipped with norm $\|y\|_{B C^{\lambda}(J ; U)}:=$ $\sup _{t \in J}\|y(t)\|_{U} e^{\lambda t}$. In case of $\lambda=0$ we simply write $B C(J ; U)$ for $B C^{0}(J ; U)$, the space of bounded continuous functions on $J$ with supremum norm. Obviously, if $J=\boldsymbol{R}^{+}$and $\lambda \geq \lambda^{\prime}$, we have the inclusion map $B C^{\lambda}(J ; U) \hookrightarrow$ $B C^{\lambda^{\prime}}(J ; U)$ and the estimate

$$
\|y\|_{B C^{\lambda^{\prime}(J ; U)}} \leq\|y\|_{B C^{\lambda}(J ; U)} \quad \text { for } y \in B C^{\lambda}(J ; U) ;
$$

to the contrary, in case of $J=\boldsymbol{R}^{-}$, the same conclusion holds if $\lambda \leq \lambda^{\prime}$.

In the following, restricting the functions with values in an open subset $\mathcal{O}$ of $U$, we often use the symbols $B C^{\lambda}(J ; \mathcal{O}), B C^{\lambda^{\prime}}(J ; \mathcal{O})$ and so on to denote open subsets of the spaces $B C^{\lambda}(J ; U), B C^{\lambda^{\prime}}(J ; U)$ and so on, respectively.

Now let $U, W$ be Banach spaces and $\mathcal{O}$ an open set of $U$, and consider a continuous mapping $h: \mathcal{O} \rightarrow W$. Then $h$ induces a mapping $\tilde{h}$ from $C(J ; \mathcal{O})$ to $C(J ; W)$ by letting

$$
[\tilde{h}(y)](t):=h(y(t)) \quad \text { for } y \in C(J ; \mathcal{O}) \text { and } t \in J .
$$

We recall the following facts on the continuity and the smoothness of $\tilde{h}$ (cf. [6, Appendix IV]), which will be employed in the subsequent sections: Assume that $h$ is of class $C^{l}, l=0,1,2, \ldots$, and let $\lambda>0$. Then $\widetilde{D^{p} h}: C\left(\boldsymbol{R}^{+} ; \mathcal{O}\right) \rightarrow$ $C\left(\boldsymbol{R}^{+} ; \mathscr{L}^{p}(U ; W)\right)$, the induced map by $D^{p} h$, defines a continuous mapping from $B C^{\lambda}\left(\boldsymbol{R}^{+} ; \mathcal{O}\right)$ to $B C\left(\boldsymbol{R}^{+} ; \mathscr{L}^{p}(U ; W)\right)$ for $p=0,1, \ldots, l$, where we promise $\mathscr{L}^{0}(U ; W):=W$ for convenience. On the other hand, assume that $h$ is of class $C^{l}$ with $\sup _{u \in \mathcal{O}}\left\|D^{p} h(u)\right\|_{\mathscr{L}^{p}(U ; W)}<\infty, p=0,1, \ldots, l$, and that $\lambda \geq 0$, then $\tilde{h}$ defines a $C^{l}$-mapping from $B C^{\lambda}\left(\boldsymbol{R}^{-} ; \mathcal{O}\right)$ to $B C^{\lambda^{\prime}}\left(\boldsymbol{R}^{-} ; W\right)$ provided that $\lambda^{\prime}>l \lambda$ (If we regard $\tilde{h}$ as a map from $B C^{\lambda}\left(\boldsymbol{R}^{-} ; \mathcal{O}\right)$ to $B C^{\lambda}\left(\boldsymbol{R}^{-} ; W\right)$, then even its $C^{1}$-smoothness is not guaranteed in general). In addition

$$
\left[\left[D^{p} \tilde{h}(y)\right] u\right](t)=D^{p} h(y(t)) u(t), \quad p=0,1, \ldots, l
$$

holds for $y \in B C^{\lambda}\left(\boldsymbol{R}^{-} ; \mathcal{O}\right), u \in\left(B C^{\lambda}\left(\boldsymbol{R}^{-} ; U\right)\right)^{\times p}$, the $p$-copies of $B C^{\lambda}\left(\boldsymbol{R}^{-} ; U\right)$, and $t \in \boldsymbol{R}^{-}$; in other words $D^{p} \tilde{h}(y)=\widetilde{D^{p} h}(y)$ holds, where $\widetilde{D^{p} h}(y)$ is interpreted as the bounded $p$-linear map from $B C^{\lambda}\left(\boldsymbol{R}^{-} ; U\right)$ to $B C^{\lambda}\left(\boldsymbol{R}^{-} ; W\right)$ given by

$$
\left.\widetilde{D^{p} h}(y) u\right](t):=D^{p} h(y(t)) u(t) \quad \text { for } t \in \boldsymbol{R}^{-} \text {. }
$$

Let $\lambda$ be a real number, $p$ a positive integer and $J$ an interval. Given $Q \in \mathscr{L}^{p}\left(U ; B C^{\lambda}(J ; W)\right)$, set

$$
[\hat{Q}(t)]\left(u_{1}, \ldots, u_{p}\right):=Q\left(u_{1}, \ldots, u_{p}\right)(t) \quad \text { for } u_{j} \in U(j=1, \ldots, p) \text { and } t \in J .
$$


Then, it is easy to see that $\hat{Q}$ belongs to $B C^{\lambda}\left(J ; \mathscr{L}^{p}(U ; W)\right)$ and that $\mathscr{L}^{p}\left(U ; B C^{\lambda}(J ; W)\right)$ is isomorphic to $B C^{\lambda}\left(J ; \mathscr{L}^{p}(U ; W)\right)$ by assigning $Q$ to $\hat{Q}$, and moreover

$$
\|\hat{Q}\|_{B C^{\lambda}\left(J ; \mathscr{L}^{p}(U ; W)\right)}=\|Q\|_{\mathscr{L}^{p}\left(U ; B C^{\lambda}(J ; W)\right)} .
$$

So we will identify these spaces and use, for abbreviation, the notation $\|\cdot\|_{\lambda, \mathscr{L}^{p}(U ; W)}$ instead of $\|\cdot\|_{B C^{\lambda}\left(J ; \mathscr{L}^{p}(U ; W)\right)}$.

Let $\boldsymbol{J}=\boldsymbol{Z}^{+}$or $\boldsymbol{Z}^{-}$. Similar results are valid for Banach spaces

$$
B^{\lambda}(J ; U):=\left\{y: J \rightarrow U: \sup _{n \in J}\|y(n)\|_{U} e^{\lambda n}<\infty\right\}, \quad \lambda \geq 0
$$

with norm $\|y\|_{B^{\lambda}(J ; U)}:=\sup _{n \in J}\|y(n)\|_{U} e^{\lambda n}$, and a continuous mapping $h: \mathcal{O} \subset U \rightarrow W$; we omit the details.

We also use the notation $B_{U}(\rho)$ which stands for the open ball in a Banach space $U$ at the center 0 with radius $\rho>0$, i.e., $B_{U}(\rho)=\left\{u \in U:\|u\|_{U}<\rho\right\}$, and by $\bar{B}_{U}(\rho)$ we mean the closure of $B_{U}(\rho)$.

\section{Stable manifolds and unstable manifolds for FDE}

In this section, we shall establish the existence of smooth local stable manifolds and unstable manifolds for the functional differential equation

$$
\dot{x}(t)=A x(t)+L\left(x_{t}\right)+f\left(x_{t}\right)
$$

under the asumption that the zero solution of (4) is hyperbolic.

For $r>0$ and $\delta>0$ we set

$$
W_{\mathrm{loc}}^{s}(r, \delta)=\left\{\phi \in \mathscr{B}:\left\|\Pi^{s} \phi\right\|_{\mathscr{B}}<r,\left\|x_{t}(0, \phi ; f)\right\|_{\mathscr{B}}<\delta, t \in \boldsymbol{R}^{+}\right\} .
$$

Then $W_{\mathrm{loc}}^{s}(r, \delta)$ is called the local stable manifold for (7); in fact it is diffeomorphic to an open set in $E^{s}$ if $r>0$ and $\delta>0$ are sufficiently small, as is shown in the following theorem.

Theorem 5. Assume that the zero solution of (4) is hyperbolic and that $f \in C^{k}(\mathscr{B} ; X)$ with $f(0)=D f(0)=0$. Then there exist an $r>0$ and a $\delta>0$ such that $W_{\mathrm{loc}}^{s}(r, \delta)$ is a $C^{k}$-manifold diffeomorphic to an open set in $E^{s}$ with the properties

(i) $W_{\text {loc }}^{s}(r, \delta)$ is tangent to $E^{s}$ at zero,

(ii) to any $\beta \in(0, \alpha)$ there corresponds a positive constant $M$ such that

$$
\left\|x_{t}(0, \phi ; f)\right\|_{\mathscr{B}} \leq M e^{-\beta t}\|\phi\|_{\mathscr{B}}, \quad t \in \boldsymbol{R}^{+}, \phi \in W_{\mathrm{loc}}^{s}(r, \delta),
$$

(iii) $W_{\mathrm{loc}}^{s}(r, \delta)$ is locally positively invariant for (7), that is, if $\phi \in W_{\mathrm{loc}}^{s}(r, \delta)$, we have $x_{\tau}(0, \phi ; f) \in W_{\mathrm{loc}}^{s}(r, \delta)$ for $\tau \in \boldsymbol{R}^{+}$whenever $\Pi^{s} x_{\tau}(0, \phi ; f) \in$ $B_{E^{s}}(r)$. 
In the proof of Theorem 5 the key is Lemma 1 in [29], which asssumes the hyperbolicity of the zero solution of Eq. (4). We will, however, state here its slight modification to the general case where the zero solution is not necessarily hyperbolic:

Lemma 1. Let $\psi \in E^{c s}$, and $\eta$ be a constant less than $\alpha$. If $x$ is a solution of Eq. (7) with the properties that $\Pi^{c s} x_{0}=\psi$, $\sup _{t \geq 0}\left\|x_{t}\right\|_{\mathscr{B}} e^{-\eta t}<\infty$ and $\sup _{t \geq 0}\left\|f\left(x_{t}\right)\right\|_{X}<\infty$, then

$$
\begin{aligned}
x_{t}= & V(t) \psi+\lim _{n \rightarrow \infty} \int_{0}^{t} V(t-s) \Pi^{c s} \Gamma^{n} f\left(x_{s}\right) d s \\
& -\lim _{n \rightarrow \infty} \int_{t}^{\infty} V(t-s) \Pi^{u} \Gamma^{n} f\left(x_{s}\right) d s, \quad t \in \boldsymbol{R}^{+} .
\end{aligned}
$$

Conversely, if $\quad y \in C\left(\boldsymbol{R}^{+} ; \mathscr{B}\right) \quad$ with $\quad \sup _{t \geq 0}\|y(t)\|_{\mathscr{B}} e^{-\eta t}<\infty \quad$ and $\sup _{t \geq 0}\|f(y(t))\|_{X}<\infty$ satisfies

$$
\begin{aligned}
y(t)= & V(t) \psi+\lim _{n \rightarrow \infty} \int_{0}^{t} V(t-\tau) \Pi^{s} \Gamma^{n} f(y(s)) d \tau \\
& -\lim _{n \rightarrow \infty} \int_{t}^{\infty} V(t-\tau) \Pi^{u} \Gamma^{n} f(y(s)) d \tau, \quad t \in \boldsymbol{R}^{+},
\end{aligned}
$$

then the function $\xi(t)$ defined by

$$
\xi(t):= \begin{cases}{[y(t)](0),} & t \geq 0, \\ {[y(0)](t),} & t<0\end{cases}
$$

is a solution of (7) satisfying $\Pi^{c s} \xi_{0}=\psi, \sup _{t \geq 0}\left\|\xi_{t}\right\|_{\mathscr{B}} e^{-\eta t}<\infty$ and $\xi_{t}=y(t)$ for $t \in \boldsymbol{R}^{+}$.

Proof. We first prove the former part of the lemma. Let $h(t)=f\left(x_{t}\right)$ for $t \geq 0$. Then $h$ belongs to $B C\left(\boldsymbol{R}^{+} ; X\right)$ and $x$ is a solution of Eq. (3) on $\boldsymbol{R}^{+}$. It follows from Theorem 1 that

$$
x_{t}=V(t-\sigma) x_{\sigma}+\lim _{n \rightarrow \infty} \int_{\sigma}^{t} V(t-s) \Gamma^{n} h(s) d s, \quad t \geq \sigma \geq 0,
$$

so that

$$
\Pi^{c s} x_{t}=V(t-\sigma) \Pi^{c s} x_{\sigma}+\lim _{n \rightarrow \infty} \int_{\sigma}^{t} V(t-s) \Pi^{c s} \Gamma^{n} h(s) d s
$$

and hence

$$
\Pi^{c s} x_{t}=V(t) \psi+\lim _{n \rightarrow \infty} \int_{0}^{t} V(t-s) \Pi^{c s} \Gamma^{n} h(s) d s, \quad t \geq 0 .
$$


We also have

$$
\Pi^{u} x_{t}=V(t-\sigma) \Pi^{u} x_{\sigma}+\lim _{n \rightarrow \infty} \int_{\sigma}^{t} V(t-s) \Pi^{u} \Gamma^{n} h(s) d s,
$$

and in particular

$$
\begin{aligned}
\Pi^{u} x_{\sigma} & =V(\sigma-t)\left[\Pi^{u} x_{t}-\lim _{n \rightarrow \infty} \int_{\sigma}^{t} V(t-s) \Pi^{u} \Gamma^{n} h(s) d s\right], \\
& =V(\sigma-t) \Pi^{u} x_{t}-\lim _{n \rightarrow \infty} \int_{\sigma}^{t} V(\sigma-s) \Pi^{u} \Gamma^{n} h(s) d s, \quad t \geq \sigma \geq 0 .
\end{aligned}
$$

Noting that

$$
\left\|V(\sigma-t) \Pi^{u} x_{t}\right\|_{\mathscr{B}} \leq C C_{1} e^{\alpha(\sigma-t)} e^{\eta t}\left(\sup _{t \geq 0}\left\|x_{t}\right\|_{\mathscr{B}} e^{-\eta t}\right) \rightarrow 0 \quad \text { as } t \rightarrow \infty,
$$

we get

$$
\Pi^{u} x_{\sigma}=-\lim _{t \rightarrow \infty} \lim _{n \rightarrow \infty} \int_{\sigma}^{t} V(\sigma-s) \Pi^{u} \Gamma^{n} h(s) d s, \quad \sigma \geq 0 .
$$

On the other hand, it follows from $\left\|\Gamma^{n} x\right\|_{\mathscr{B}} \leq K\|x\|_{X}$ that for $t_{2} \geq t_{1} \geq \sigma$

$$
\left\|\int_{t_{1}}^{t_{2}} V(\sigma-s) \Pi^{u} \Gamma^{n} h(s) d s\right\|_{\mathscr{B}} \leq\left(C C_{1} K / \alpha\right)\left(\sup _{t \geq 0}\|h(t)\|_{X}\right) e^{\alpha\left(\sigma-t_{1}\right)} \rightarrow 0
$$

as $t_{1} \rightarrow \infty$. Consequently, the convergence in $\mathscr{B}$ of

$$
\lim _{t \rightarrow \infty} \int_{\sigma}^{t} V(\sigma-s) \Pi^{u} \Gamma^{n} h(s) d s=\int_{\sigma}^{\infty} V(\sigma-s) \Pi^{u} \Gamma^{n} h(s) d s
$$

is uniform for $n$. On the other hand, $\left\{\int_{\sigma}^{\infty} V(\sigma-s) \Pi^{u} \Gamma^{n} h(s) d s\right\}_{n \geq 1}$ is a Cauchy sequence in $\mathscr{B}$ since

$$
\begin{aligned}
\limsup _{n, m \rightarrow \infty}\left\|\int_{\sigma}^{\infty} V(\sigma-s) \Pi^{u} \Gamma^{n} h(s) d s-\int_{\sigma}^{\infty} V(\sigma-s) \Pi^{u} \Gamma^{m} h(s) d s\right\|_{\mathscr{B}} \\
\leq\left(2 C C_{1} K / \alpha\right)\left(\sup _{t \geq 0}\|h(t)\|_{X}\right) e^{\alpha(\sigma-t)} \\
\quad+\limsup _{n, m \rightarrow \infty}\left\|\Pi^{u}\left(\int_{\sigma}^{t} V(\sigma-s) \Gamma^{n} h(s) d s-\int_{\sigma}^{t} V(\sigma-s) \Gamma^{m} h(s) d s\right)\right\|_{\mathscr{B}} \\
=\left(2 C C_{1} K / \alpha\right)\left(\sup _{t \geq 0}\|h(t)\|_{X}\right) e^{\alpha(\sigma-t)}
\end{aligned}
$$

for arbitrary $t>\sigma$. These observations yield 


$$
\begin{aligned}
\lim _{n \rightarrow \infty} \int_{\sigma}^{\infty} V(\sigma-s) \Pi^{u} \Gamma^{n} h(s) & =\lim _{n \rightarrow \infty} \lim _{t \rightarrow \infty} \int_{\sigma}^{t} V(\sigma-s) \Pi^{u} \Gamma^{n} h(s) d s \\
& =\lim _{t \rightarrow \infty} \lim _{n \rightarrow \infty} \int_{\sigma}^{t} V(\sigma-s) \Pi^{u} \Gamma^{n} h(s) d s \\
& =-\Pi^{u} x_{\sigma}, \quad \sigma \geq 0 .
\end{aligned}
$$

Thus we arrive at

$$
\begin{aligned}
x_{t} & =\Pi^{c s} x_{t}+\Pi^{u} x_{t} \\
& =V(t) \psi+\lim _{n \rightarrow \infty} \int_{0}^{t} V(t-s) \Pi^{c s} \Gamma^{n} h(s) d s-\lim _{n \rightarrow \infty} \int_{t}^{\infty} V(t-s) \Pi^{u} \Gamma^{n} h(s) d s, \quad t \geq 0,
\end{aligned}
$$

as required.

We next prove the latter part of the lemma. Set $g(t)=f(y(t))$ for $t \geq 0$. Then $g$ belongs to $B C\left(\boldsymbol{R}^{+} ; X\right)$, and by the same argument as the former part of the lemma, the limit $\lim _{n \rightarrow \infty} \int_{t}^{\infty} V(t-s) \Pi^{u} \Gamma^{n} g(s) d s$ exists for each $t \geq 0$. Let us denote by $v(t)$ the solution of Eq. (3) with $h=g$ on $\boldsymbol{R}^{+}$with $v_{0}=y(0)$. Then Theorem 1 assures

$$
\begin{aligned}
v_{t} & =V(t) y(0)+\lim _{n \rightarrow \infty} \int_{0}^{t} V(t-s) \Gamma^{n} g(s) d s \\
& =V(t)\left\{\psi-\lim _{n \rightarrow \infty} \int_{0}^{\infty} V(-s) \Pi^{u} \Gamma^{n} g(s) d s\right\}+\lim _{n \rightarrow \infty} \int_{0}^{t} V(t-s) \Gamma^{n} g(s) d s \\
& =V(t) \psi-\lim _{n \rightarrow \infty} \int_{0}^{\infty} V(t-s) \Pi^{u} \Gamma^{n} g(s) d s+\lim _{n \rightarrow \infty} \int_{0}^{t} V(t-s)\left(\Pi^{c s}+\Pi^{u}\right) \Gamma^{n} g(s) d s \\
& =V(t) \psi+\lim _{n \rightarrow \infty} \int_{0}^{t} V(t-s) \Pi^{c s} \Gamma^{n} g(s) d s-\lim _{n \rightarrow \infty} \int_{t}^{\infty} V(t-s) \Pi^{u} \Gamma^{n} g(s) d s \\
& =y(t)
\end{aligned}
$$

for $t \geq 0$. In particular, it follows that

$$
v(t)=[y(t)](0)=\xi(t), \quad t \geq 0,
$$

so that $\xi$ is continuous on $\boldsymbol{R}^{+}$. Hence, in view of $\left(A_{1}\right)$, it turns out that $\xi_{t} \in \mathscr{B}$, and that $\xi_{t}=y(t)$ for all $t \geq 0$ since

$$
\begin{aligned}
\left\|\xi_{t}-y(t)\right\|_{\mathscr{B}} & =\left\|\xi_{t}-v_{t}\right\|_{\mathscr{B}} \\
& \leq K \sup _{0 \leq s \leq t}\|\xi(s)-v(s)\|_{X}+M(t)\left\|\xi_{0}-y(0)\right\|_{\mathscr{B}} \\
& =0
\end{aligned}
$$


In particular, it follows that $\sup _{t \geq 0}\left\|\xi_{t}\right\|_{\mathscr{B}} e^{-\eta t}=\sup _{t \geq 0}\|y(t)\|_{\mathscr{B}} e^{-\eta t}<\infty$. Also

$$
\Pi^{c s} \xi_{0}=\Pi^{c s} y(0)=\Pi^{c s}\left(\psi-\lim _{n \rightarrow \infty} \int_{0}^{\infty} V(-s) \Pi^{u} \Gamma^{n} h(s) d s\right)=\psi .
$$

Noting that $\xi(t)=v(t)=x\left(t, 0, \xi_{0} ; g\right)$ and $g(t)=f(y(t))=f\left(\xi_{t}\right)$ for $t \geq 0$, we see that $\xi$ is a solution of Eq. (7) on $\boldsymbol{R}^{+}$. The proof is completed.

We are now in a position to give the proof of Theorem 5.

Proof of Theorem 5. Since $f \in C^{k}(\mathscr{B} ; X)$ with $f(0)=D f(0)=0$, there exist a constant $\delta_{0}>0$ and a nondecreasing function $\zeta:\left[0, \delta_{0}\right] \rightarrow \boldsymbol{R}^{+}$such that $\zeta(0)=0$ and

$$
\|f(\phi)-f(\psi)\|_{X} \leq \zeta(\delta)\|\phi-\psi\|_{\mathscr{B}}, \quad \phi, \psi \in B_{\mathscr{B}}(\delta)
$$

for $\delta \in\left[0, \delta_{0}\right]$. Let $\beta$ be a positive number less than $\alpha$, and $Y$ the Banach space $B C^{\beta}\left(\boldsymbol{R}^{+} ; \mathscr{B}\right)$. Given $r>0$ and $\delta \in\left(0, \delta_{0}\right]$, set

$$
\begin{aligned}
\mathscr{F}(\psi, y)(t):= & y(t)-V(t) \psi-\lim _{n \rightarrow \infty} \int_{0}^{t} V(t-s) \Pi^{s} \Gamma^{n} f(y(s)) d s \\
& +\lim _{n \rightarrow \infty} \int_{t}^{\infty} V(t-s) \Pi^{u} \Gamma^{n} f(y(s)) d s
\end{aligned}
$$

for $(\psi, y) \in B_{E^{s}}(r) \times B_{Y}(\delta)$ and $t \in \boldsymbol{R}^{+}$. Then $\mathscr{F}$ defines a map from $\mathscr{F}: B_{E^{s}}(r) \times B_{Y}(\delta)$ to $Y$. Indeed, since

$$
\begin{aligned}
\left\|\lim _{n \rightarrow \infty} \int_{0}^{t} V(t-s) \Pi^{s} \Gamma^{n} f(y(s)) d s\right\|_{\mathscr{B}} & \leq C C_{1} K \zeta(\delta) \int_{0}^{t} e^{-\alpha(t-s)}\|y\|_{Y} e^{-\beta s} d s \\
& \leq\left(C C_{1} K \zeta(\delta) /(\alpha-\beta)\right)\|y\|_{Y} e^{-\beta t}
\end{aligned}
$$

and also

$$
\begin{aligned}
\left\|\lim _{n \rightarrow \infty} \int_{t}^{\infty} V(t-s) \Pi^{u} \Gamma^{n} f(y(s)) d s\right\|_{\mathscr{B}} & \leq C C_{1} K \zeta(\delta) \int_{t}^{\infty} e^{\alpha(t-s)}\|y\|_{Y} e^{-\beta s} d s \\
& =\left(C C_{1} K \zeta(\delta) /(\alpha+\beta)\right)\|y\|_{Y} e^{-\beta t}
\end{aligned}
$$

for $t \in \boldsymbol{R}^{+}$, it follows that $\|\mathscr{F}(\psi, y)(t)\|_{\mathscr{B}} \leq\left(C r+\left(\delta+c_{*}(\beta) \zeta(\delta)\right)\|y\|_{Y}\right) e^{-\beta t}$ for $t \in \boldsymbol{R}^{+}$, where $c_{*}(\beta):=C C_{1} K(1 /(\alpha-\beta)+1 /(\alpha+\beta))$. Hence $\mathscr{F}$ is well-defined. Moreover, $\mathscr{F}$ is of class $C^{k}$. Let us check this below. It is obvious that if we set $\mathscr{J}(\psi, y):=y-V(\cdot) \psi$, then $D_{\psi} \mathscr{J}(\psi, y)=-V(\cdot)$ and $D_{y} \mathscr{J}(\psi, y)=I_{Y}$, where $D_{\psi} \mathscr{J}$ and $D_{y} \mathscr{J}$ stands for the derivatives of $\mathscr{J}$ in the variables $\psi$ and $y$, respectively; and therefore $\mathscr{J}$ is a $C^{k}$-mapping from $B_{E^{s}}(r) \times B_{Y}(\delta)$ to $Y$. So, it suffices to prove the $C^{k}$-smoothness of $\mathscr{K}: B_{Y}(\delta) \rightarrow Y$ defined by 


$$
\mathscr{K}(y)(t):=\lim _{n \rightarrow \infty} \int_{0}^{t} V(t-s) \Pi^{s} \Gamma^{n} f(y(s)) d s-\lim _{n \rightarrow \infty} \int_{t}^{\infty} V(t-s) \Pi^{u} \Gamma^{n} f(y(s)) d s .
$$

Let us define a map $A: B_{Y}(\delta) \rightarrow \mathscr{L}(Y)$ by setting

$$
\begin{aligned}
{[A(y) u](t):=} & \lim _{n \rightarrow \infty} \int_{0}^{t} V(t-s) \Pi^{s} \Gamma^{n}(D f(y(s)) u(s)) d s \\
& -\lim _{n \rightarrow \infty} \int_{t}^{\infty} V(t-s) \Pi^{u} \Gamma^{n}(D f(y(s)) u(s)) d s
\end{aligned}
$$

for $u \in Y$ and $t \in \boldsymbol{R}^{+}$. It then follows that

$$
\begin{aligned}
\|[A(y) u](t)\|_{\mathscr{B}} e^{\beta t}= & e^{\beta t}\left\|\lim _{n \rightarrow \infty} \int_{0}^{t} V(t-s) \Pi^{s} \Gamma^{n}(D f(y(s)) u(s)) d s\right\|_{\mathscr{B}} \\
& +e^{\beta t}\left\|\lim _{n \rightarrow \infty} \int_{t}^{\infty} V(t-s) \Pi^{u} \Gamma^{n}(D f(y(s)) u(s)) d s\right\|_{\mathscr{B}} \\
\leq & C C_{1} K\|\widetilde{D f}(y)\|_{0, \mathscr{L}(\mathscr{B} ; X)}\|u\|_{Y} e^{\beta t} \\
& \times\left(\int_{0}^{t} e^{-\alpha(t-s)} e^{-\beta s} d s+\int_{t}^{\infty} e^{\alpha(t-s)} e^{-\beta s} d s\right) \\
= & c_{*}(\beta)\|\widetilde{D f}(y)\|_{0, \mathscr{L}(\mathscr{B} ; X)}\|u\|_{Y}, \quad t \geq 0,
\end{aligned}
$$

where $\widetilde{D f}$ is the induced map by $D f$ (section 2.4), and therefore from (8) that

$$
\|A(y) u\|_{Y} \leq c_{*}(\beta)\|\widetilde{D f}(y)\|_{0, \mathscr{L}(\mathscr{B} ; X)}\|u\|_{Y} \leq c_{*}(\beta) \zeta(\delta)\|u\|_{Y}
$$

Hence $A(y) \in \mathscr{L}(Y)$, and $A$ is well-defined. Similarly, for $h \in Y$ with $\|h\|_{Y}$ small, we see

$$
\|A(y+h) u-A(y) u\|_{Y} \leq c_{*}(\beta)\|\widetilde{D f}(y+h)-\widetilde{D f}(y)\|_{0, \mathscr{L}(\mathscr{B} ; X)}\|u\|_{Y},
$$

and in particular

$$
\|A(y+h)-A(y)\|_{\mathscr{L}(Y)} \leq c_{*}(\beta)\|\widetilde{D f}(y+h)-\widetilde{D f}(y)\|_{0, \mathscr{L}(\mathscr{B} ; X)} .
$$

Thus, the continuity of $A$ follows from that of $\widetilde{D f}: Y \rightarrow B C\left(\boldsymbol{R}^{+} ; \mathscr{L}(\mathscr{B} ; X)\right)$. Noting

$$
\begin{aligned}
\mathscr{K}(y+u)-\mathscr{K}(y)-A(y) u \\
=\lim _{n \rightarrow \infty} \int_{0}^{t} V(t-s) \Pi^{s} \Gamma^{n}\left(\int_{0}^{1}(D f(y(s)+\sigma u(s))-D f(y(s))) u(s) d \sigma\right) d s \\
\quad+\lim _{n \rightarrow \infty} \int_{t}^{\infty} V(t-s) \Pi^{u} \Gamma^{n}\left(\int_{0}^{1}(D f(y(s)+\sigma u(s))-D f(y(s))) u(s) d \sigma\right) d s,
\end{aligned}
$$


we get analogously to (9) that

$$
\begin{aligned}
& \|\mathscr{K}(y+u)-\mathscr{K}(y)-A(y) u\|_{Y} \\
& \quad \leq c_{*}(\beta) \sup _{0 \leq \sigma \leq 1}\|\widetilde{D f}(y+\sigma u)-\widetilde{D f}(y)\|_{0, \mathscr{L}(\mathscr{B} ; X)}\|u\|_{Y},
\end{aligned}
$$

which yields $\lim _{u \rightarrow 0}\|\mathscr{K}(y+u)-\mathscr{K}(y)-A(y) u\|_{Y} /\|u\|_{Y}=0$. So $D \mathscr{K}(y)=$ $A(y)$, and hence $\mathscr{K}$ is of class $C^{1}$. Similarly in virtue of the continuity of $\widehat{D^{p} f}: Y \rightarrow B C\left(\boldsymbol{R}^{+} ; \mathscr{L}^{p}(\mathscr{B} ; X)\right), 1 \leq p \leq k$, we find that

$$
\begin{aligned}
{\left[D^{p} \mathscr{K}(y) w\right](t)=} & \lim _{n \rightarrow \infty} \int_{0}^{t} V(t-s) \Pi^{s} \Gamma^{n}\left(D^{p} f(y(s)) w(s)\right) d s \\
& -\lim _{n \rightarrow \infty} \int_{t}^{\infty} V(t-s) \Pi^{u} \Gamma^{n}\left(D^{p} f(y(s)) w(s)\right) d s
\end{aligned}
$$

for $w \in Y^{\times p}, p=1, \ldots, k$ and $t \in \boldsymbol{R}^{+}$. Consequently, $\mathscr{K}$, and hence $\mathscr{F}$ are of class $C^{k}$.

The observation above shows in particular

$$
\left[D_{\psi} \mathscr{F}(\psi, y) \phi\right](t)=-V(t) \phi, \quad \phi \in E^{s} .
$$

and

$$
\begin{aligned}
{\left[D_{y} \mathscr{F}(\psi, y) u\right](t)=} & u(t)-\lim _{n \rightarrow \infty} \int_{0}^{t} V(t-\tau) \Pi^{s} \Gamma^{n}(D f(y(\tau)) u(\tau)) d \tau \\
& +\lim _{n \rightarrow \infty} \int_{t}^{\infty} V(t-\tau) \Pi^{u} \Gamma^{n}(D f(y(\tau)) u(\tau)) d \tau, \quad u \in Y .
\end{aligned}
$$

Since $D_{y} \mathscr{F}(0,0)=I_{Y}$, the implicit function theorem (e.g., see [23, Theorem 5.9 (p. 19) in Chapter I]) implies that for small $r>0$ there exists a unique $C^{k}$-mapping $\Lambda: B_{E^{s}}(r) \rightarrow B_{Y}(\delta)$ such that $\Lambda(0)=0$ and $\mathscr{F}(\psi, \Lambda(\psi))=0$ for $\psi \in B_{E^{s}}(r)$, or equivalently,

$$
\begin{aligned}
\Lambda(\psi)(t)= & V(t) \psi+\lim _{n \rightarrow \infty} \int_{0}^{t} V(t-s) \Pi^{s} \Gamma^{n} f(\Lambda(\psi)(s)) d s \\
& -\lim _{n \rightarrow \infty} \int_{t}^{\infty} V(t-s) \Pi^{u} \Gamma^{n} f(\Lambda(\psi)(s)) d s
\end{aligned}
$$

for $t \in \boldsymbol{R}^{+}$. In particular, we get

$$
\Lambda(\psi)(0)=\psi-\lim _{n \rightarrow \infty} \int_{0}^{\infty} V(-\tau) \Pi^{u} \Gamma^{n} f(\Lambda(\psi)(\tau)) d \tau, \quad \psi \in B_{E^{s}}(r) .
$$

Now define a mapping $H: B_{E^{s}}(r) \rightarrow E^{u}$ by letting 


$$
H(\psi)=-\lim _{n \rightarrow \infty} \int_{0}^{\infty} V(-\tau) \Pi^{u} \Gamma^{n} f(\Lambda(\psi)(\tau)) d \tau .
$$

Notice that $H$ is also of class $C^{k}$ since $H=\Pi^{u} \circ \mathrm{ev}_{0} \circ \Lambda$, where $\mathrm{ev}_{0}: Y \rightarrow X$ is the mapping given by $\operatorname{ev}_{0}(y):=y(0)$ for $y \in Y$.

Properties (i) through (iii) are confirmed as follows. We first claim that graph $H=W_{\text {loc }}^{s}(r, \delta), r>0$ being the one in the above, which implies that $W_{\text {loc }}^{s}(r, \delta)$ is a $C^{k}$-manifold diffeomorphic to $B_{E^{s}}(r)$. Indeed, graph $H \subset$ $W_{\text {loc }}^{s}(r, \delta)$ is obvious. Conversely, let $\phi \in W_{\text {loc }}^{s}(r, \delta)$. Then, by virtue of Lemma 1 (with $\eta=0$ ) and the unique existence of $\Lambda(\psi)$, we obtain $x_{t}(0, \phi ; f)=\Lambda(\psi)(t)$ with $\psi=\Pi^{s} \phi$ for $t \in \boldsymbol{R}^{+}$; and hence $\phi=\psi+H(\psi) \in$ graph $H$. Thus, Property (i) holds true if it is shown that graph $H$ is tangent to $E^{s}$ at $\psi=0$. For this it is sufficient to verify that $D H(0)=0$. In view of the relation $\mathscr{F}(\psi, \Lambda(\psi))=0$, we get

$$
D_{\psi} \mathscr{F}(\psi, \Lambda(\psi))+D_{y} \mathscr{F}(\psi, \Lambda(\psi)) D \Lambda(\psi)=0,
$$

and in particular $D \Lambda(0)=-D_{\psi} \mathscr{F}(0,0)$. Then it follows from (10) that

$$
D \Lambda(0) \phi=-D_{\psi} \mathscr{F}(0,0) \phi=V(\cdot) \phi \quad \text { for } \phi \in E^{s} .
$$

Consequently,

$$
D H(0) \phi=\left(\Pi^{u} \circ \mathrm{ev}_{0} \circ D \Lambda\right)(0) \phi=\Pi^{u}(V(0) \phi)=\Pi^{u} \phi=0 \quad \text { for } \phi \in E^{s},
$$

i.e., $D H(0)=0$, as desired.

(ii) Let $\phi \in W_{\mathrm{loc}}^{s}(r, \delta)$. Then, by Property (i), $\phi=\psi+H(\psi)$ with $\psi:=$ $\Pi^{s} \phi$. By virtue of Eq. (11) and the fact that $\sup _{t \in \boldsymbol{R}^{+}}\|f(\Lambda(\psi)(t))\|_{X}<\infty$, it follows from Lemma 1 that

$$
\begin{aligned}
\Lambda(\psi)(t) & =x_{t}(0, \Lambda(\psi)(0) ; f)=x_{t}(0, \psi+H(\psi) ; f) \\
& =x_{t}(0, \phi ; f) \quad \text { for } t \in \boldsymbol{R}^{+} .
\end{aligned}
$$

Since $\mathscr{F}$ is of class $C^{k}$ and $D_{y} \mathscr{F}(0,0)=I_{Y}$, by taking $r>0$ and $\delta>0$ small enough, we may assume that

$$
\left\|D_{y} \mathscr{F}(\psi, y)^{-1}\right\|_{\mathscr{L}(Y)} \leq K_{1}, \quad(\psi, y) \in B_{E^{s}}(r) \times B_{Y}(\delta)
$$

for some constant $K_{1}>0$. In view of (10) and (12), we get

$$
\begin{aligned}
\|\Lambda(\psi)\|_{Y} & \leq \int_{0}^{1}\|D \Lambda(t \psi) \psi\|_{\mathscr{L}\left(E^{s} ; Y\right)} d t \\
& \leq \int_{0}^{1}\left\|D_{y} \mathscr{F}(t \psi, \Lambda(t \psi))^{-1}\right\|_{\mathscr{L}(Y)}\left\|D_{\psi} \mathscr{F}(t \psi, \Lambda(t \psi)) \psi\right\|_{\mathscr{L}\left(E^{s} ; Y\right)} d t \\
& \leq K_{1}\|V(\cdot) \psi\|_{Y} \leq C K_{1}\|\psi\|_{\mathscr{B}},
\end{aligned}
$$


and therefore combined with (14)

$$
\left\|x_{t}(0, \phi ; f)\right\|_{\mathscr{B}}=\|\Lambda(\psi)(t)\|_{\mathscr{B}} \leq\|\Lambda(\psi)\|_{Y} e^{-\beta t} \leq C K_{1}\|\psi\|_{\mathscr{B}} e^{-\beta t} .
$$

On the other hand, since $D H(0)=0$, there is an $a \in(0,1)$ such that $\|H(\psi)\|_{\mathscr{B}} \leq$ $a\|\psi\|_{\mathscr{B}}$ for $\psi \in B_{E^{s}}(r)$ with $r>0$ small. Hence we obtain $\|\phi\|_{\mathscr{B}} \geq\|\psi\|_{\mathscr{B}}-$ $\|H(\psi)\|_{\mathscr{B}} \geq(1-a)\|\psi\|_{\mathscr{B}}$, and together with (15)

$$
\left\|x_{t}(0, \phi ; f)\right\|_{\mathscr{B}} \leq \frac{C K_{1}}{1-a}\|\phi\|_{\mathscr{B}} e^{-\beta t} \quad \text { for } \phi \in W_{\mathrm{loc}}^{s}(r, \delta), t \in \boldsymbol{R}^{+} .
$$

Consequently, (ii) holds with $M=C K_{1} /(1-a)$.

(iii) Given $\psi \in B_{E^{s}}(r)$ and $\tau \in \boldsymbol{R}^{+}$, suppose that $\chi:=\Pi^{s} x_{\tau}(0, \psi+H(\psi) ; f)$ belongs to $B_{E^{s}}(r)$. Since $v(t):=x(t+\tau, 0, \psi+H(\psi) ; f)$ is a solution of Eq. (7) with $v_{t}=\Lambda(\psi)(t+\tau)$ for $t \in \boldsymbol{R}^{+}$, it follows from Lemma 1, together with the uniqueness of zeros of $\mathscr{F}(\psi, \cdot)$, that $v_{t}=\Lambda(\chi)(t)$ for $t \in \boldsymbol{R}^{+}$. In particular

$$
x_{\tau}(0, \psi+H(\psi) ; f)=v_{0}=\Lambda(\chi)(0)=\chi+H(\chi) \in \operatorname{graph} H,
$$

so that $W_{\mathrm{loc}}^{s}(r, \delta)$ is positively invariant. This completes the proof.

We shall next establish the existence of local unstable manifolds for Eq. (7). For $r>0$ and $\delta>0$ we consider the set

$$
W_{\mathrm{loc}}^{u}(r, \delta)=\left\{\phi \in \mathscr{B}:\left\|\Pi^{u} \phi\right\|_{\mathscr{B}}<r,\left\|x_{t}(0, \phi ; f)\right\|_{\mathscr{B}}<\delta, t \in \boldsymbol{R}^{-}\right\} .
$$

Then we have the following:

Theorem 6. Assume that the zero solution of (4) is hyperbolic and that $f \in C^{k}(\mathscr{B} ; X)$ with $f(0)=D f(0)=0$. Then there exist an $r>0$ and a $\delta>0$ such that $W_{\mathrm{loc}}^{u}(r, \delta)$ is a $C^{k}$-manifold diffeomorphic to an open set in $E^{u}$ with the properties

(i) $W_{\text {loc }}^{u}(r, \delta)$ is tangent to $E^{u}$ at zero,

(ii) to any $\beta \in(0, \alpha)$ there corresponds a positive constant $M$ such that

$$
\left\|x_{t}(0, \phi ; f)\right\|_{\mathscr{B}} \leq M e^{\beta t}\|\phi\|_{\mathscr{B}}, \quad t \in \boldsymbol{R}^{-}, \phi \in W_{\text {loc }}^{u}(r, \delta),
$$

(iii) $W_{\mathrm{loc}}^{u}(r, \delta)$ is locally negatively invariant for (7), that is, if $\phi \in W_{\mathrm{loc}}^{u}(r, \delta)$, we have $x_{\tau}(0, \phi ; f) \in W_{\mathrm{loc}}^{u}(r, \delta)$ for $\tau \in \boldsymbol{R}^{-}$whenever $\Pi^{u} x_{\tau}(0, \phi ; f) \in$ $B_{E^{u}}(r)$.

By means of the following lemma, one can prove the theorem in quite a similar fashion to the preceeding argument. We will omit the proof of the theorem together with that of the lemma.

Lemma 2. Let $\psi \in E^{c u}$, and $\eta$ is a constant less than $\alpha$. If $x$ is a solution of Eq. (7) with the properties that $\Pi^{c u} x_{0}=\psi$, $\sup _{t \leq 0}\left\|x_{t}\right\|_{\mathscr{B}} e^{\eta t}<\infty$, and $\sup _{t \leq 0}\left\|f\left(x_{t}\right)\right\|_{X}<\infty$, then 


$$
\begin{aligned}
x_{t}= & V(t) \psi-\lim _{n \rightarrow \infty} \int_{t}^{0} V(t-s) \Pi^{c u} \Gamma^{n} f\left(x_{s}\right) d s \\
& +\lim _{n \rightarrow \infty} \int_{-\infty}^{t} V(t-s) \Pi^{s} \Gamma^{n} f\left(x_{s}\right) d s, \quad t \in \boldsymbol{R}^{-} .
\end{aligned}
$$

Conversely, if $y \in C\left(\boldsymbol{R}^{-} ; \mathscr{B}\right)$ with $\sup _{t \leq 0}\|y(t)\|_{\mathscr{B}} e^{\eta t}<\infty$ and $\sup _{t \leq 0}\|f(y(t))\|_{X}<$ $\infty$ satisfies

$$
\begin{aligned}
y(t)= & V(t) \psi-\lim _{n \rightarrow \infty} \int_{t}^{0} V(t-s) \Pi^{c u} \Gamma^{n} f(y(s)) d s \\
& +\lim _{n \rightarrow \infty} \int_{-\infty}^{t} V(t-s) \Pi^{s} \Gamma^{n} f(y(s)) d s, \quad t \in \boldsymbol{R}^{-},
\end{aligned}
$$

then the function $\xi(t)$ defined by

$$
\xi(t):=[y(t)](0), \quad t \in \boldsymbol{R}^{-}
$$

is a solution of (7) satisfying $\Pi^{c u} \xi_{0}=\psi$, $\sup _{t \leq 0}\left\|\xi_{t}\right\|_{\mathscr{B}} e^{\eta t}<\infty$, and $\xi_{t}=y(t)$ for $t \in \boldsymbol{R}^{-}$.

\section{Center-unstable manifolds for FDE}

We discuss in this section the case where the zero solution of Eq. (4) is not necessarily hyperbolic.

Let $\left\{\phi_{1}, \ldots, \phi_{d}\right\}$ be a basis in $E^{c u}$, $d$ being the dimension of $E^{c u}$, and $\left\{\psi_{1}, \ldots, \psi_{d}\right\}$ its dual basis, where each $\psi_{j}$ is thought of as an element in $\mathscr{B}^{*}$, the dual space of $\mathscr{B}$, by the zero extension: $\left\langle\psi_{i}, \phi_{j}\right\rangle=1$ if $i=j$ and 0 otherwise, and $\left.\psi_{i}\right|_{E^{s}}=0$. Here and subsequently, $\langle$,$\rangle denotes the pairing betwen the dual$ space and the original space. Also, denote by $\Phi$ and $\Psi,\left(\phi_{1}, \ldots, \phi_{d}\right)$ and the transpose of $\left(\psi_{1}, \ldots, \psi_{d}\right)$, respectively. Then, for any $\phi \in \mathscr{B}$ the coordinate of its $E^{c u}$-component with respest to the basis $\left\{\phi_{1}, \ldots, \phi_{d}\right\}$ can be expressed by $\langle\Psi, \phi\rangle \in \boldsymbol{R}^{d}$, and in particular the projection $\Pi^{c u}$ is given by

$$
\Pi^{c u} \phi=\Phi\langle\Psi, \phi\rangle, \quad \phi \in \mathscr{B} .
$$

Since $\left\{V^{c u}(t)\right\}_{t \geq 0}$ is a strongly continuous semigroup on the finite dimensional space $E^{c u}$, there corresponds a $d \times d$-matrix $G$ with $\sigma(G)=\Sigma$ such that

$$
V^{c u}(t) \Phi=\Phi e^{t G}, \quad t \geq 0 .
$$

Then the $E^{c u}$-component of solutions of Eq. (7) can be related to those of some ordinary differential equation in $\boldsymbol{R}^{d}$; more precisely, if $x(t)$ is a solution of Eq. (7), then the function $z(t)$, given by $\Phi z(t):=\Pi^{c u} x_{t}$, is a solution to the ordinary differential equation 


$$
\dot{z}(t)=G z(t)+\left\langle x^{*}, f\left(\Phi z(t)+\Pi^{s} x_{t}\right)\right\rangle,
$$

where $x^{*}$ is a $d$-column vector in $X^{*}$ defined by

$$
\left\langle x^{*}, x\right\rangle=\lim _{n \rightarrow \infty}\left\langle\Psi, \Gamma^{n} x\right\rangle, \quad x \in X ;
$$

see [19].

For the existence of local center-unstable manifolds for Eq. (7) we have the following:

Theorem 7. Assume that $f \in C^{k}(\mathscr{B} ; X)$ with $f(0)=D f(0)=0$. Then there exist positive numbers $r, \delta$, and a $C^{k}$-mapping $H_{*}: B_{E^{c u}}(r) \rightarrow B_{E^{s}}(\delta)$ with $H_{*}(0)=0$, together with an open neighborhood $\Omega$ of 0 in $\mathscr{B}$, such that the following properties hold:

(i) $W_{\mathrm{loc}}^{c u}(r, \delta):=$ graph $H_{*}$ is tangent to $E^{c u}$ at zero,

(ii) $W_{\mathrm{loc}}^{c u}(r, \delta)$ is locally invariant for Eq. (7), that is,

(a) for any $\xi \in W_{\mathrm{loc}}^{\mathrm{cu}}(r, \delta)$ there exists a $t_{\xi}>0$ such that $x_{t}(0, \xi ; f) \in W_{\mathrm{loc}}^{c u}(r, \delta)$ for $|t| \leq t_{\xi}$,

(b) there exists an open neighborhood $\Omega$ of 0 in $\mathscr{B}$ such that if $\xi \in W_{\mathrm{loc}}^{c u}(r, \delta)$ and $x_{t}(0, \xi ; f) \in \Omega$ for $0 \leq t \leq T$, then $x_{t}(0, \xi ; f) \in$ $W_{\mathrm{loc}}^{c u}(r, \delta)$ for $0 \leq t \leq T$.

(iii) If $x$ is a solution of Eq. (7) on an interval $J$ such that $x_{t} \in W_{\mathrm{loc}}^{c u}(r, \delta)(t \in J)$, then the function $\Phi z(t):=\Pi^{c u} x_{t}$ satisfies the ordinary differential equation

$$
\dot{z}(t)=G z(t)+\left\langle x^{*}, f\left(\Phi z(t)+H_{*}(\Phi z(t))\right)\right\rangle, \quad t \in J .
$$

Conversely, if $z(t)$ satisfies the equation (17) on an interval $J$ with $\Phi z(t) \in \Omega$ on $J$, then there exists a solution $x$ of $E q$. (7) on $J$ such that $x_{t} \in W_{\mathrm{loc}}^{c u}(r, \delta)$ and $\Pi^{c u} x_{t}=\Phi z(t)$ on $J$.

We will prove the theorem by a slightly different idea from that of the proof of Theorem 5 due to the smoothness condition, mentioned in section 2.4, of $\widetilde{f}_{\delta}$, the map induced by $f_{\delta}$ defined below. We will also devide the proof of the theorem into several propositions.

Let $\rho: \boldsymbol{R} \rightarrow[0,1]$ be a $C^{\infty}$-function such that $\rho(t)=1 \quad(|t| \leq 2)$ and $\rho(t)=0(|t| \geq 3)$, and put $f_{\delta}(\phi):=\rho\left(\left\|\Pi^{s} \phi\right\|_{\mathscr{B}} / \delta\right) \rho\left(\left\|\Pi^{c u} \phi\right\|_{\mathscr{B}} / \delta\right) f(\phi)$ for $\phi \in \mathscr{B}$. Then $f_{\delta}: \mathscr{B} \rightarrow X$ is continuous on $\mathscr{B}$, and is of class $C^{k}$ when restricted to the open set $S_{\delta}:=\left\{\phi \in \mathscr{B}:\left\|\Pi^{s} \phi\right\|_{\mathscr{B}}<\delta\right\}$ since, in view of $\operatorname{dim} E^{c u}<\infty$, we may assume that $\left\|\Pi^{c u} \phi\right\|_{\mathscr{B}}$ is of class $C^{k}$ for $\phi \neq 0$. Moreover, by the assumption $f(0)=D f(0)=0$, there exisit a $\delta_{1}>0$ and a nondecreasing continuous function $\zeta_{*}:\left[0, \delta_{1}\right] \rightarrow \boldsymbol{R}^{+}$such that $\zeta_{*}(0)=0$,

$$
\left\|f_{\delta}(\phi)\right\|_{X} \leq \delta \zeta_{*}(\delta), \quad \text { and } \quad\left\|f_{\delta}(\phi)-f_{\delta}(\psi)\right\|_{X} \leq \zeta_{*}(\delta)\|\phi-\psi\|_{\mathscr{B}}
$$


for $\phi, \psi \in \mathscr{B}$ and $\delta \in\left[0, \delta_{1}\right]$. Indeed, we may put $\zeta_{*}(\delta)=$ $\left(\sup _{\|\phi\|_{\mathscr{B}} \leq 3 \delta}\|D f(\phi)\|_{\mathscr{L}(\mathscr{B} ; X)}\right) \times\left(1+3 \sup _{0 \leq t \leq 3}\left|\rho^{\prime}(t)\right|\right) \quad$ (cf. [6, Lemma 4.1]). In addition, by taking $\delta_{1}>0$ small if necessary, we may assume that there exists a positive number $M_{1}(\delta)=: M_{1}$ such that

$$
\left\|D^{p} f_{\delta}(\phi)\right\|_{\mathscr{L}^{p}(\mathscr{B} ; X)} \leq M_{1} \quad \text { for } \phi \in S_{\delta} \text { and } p=1,2, \ldots, k .
$$

Now let $\hat{\eta} \in(0, \alpha)$ be given, and choose the constant $\varepsilon$ in Theorem 2 and a positive number $\eta_{0}$ in such a way that $\varepsilon<\eta_{0}<\hat{\eta} / k$ !. Put

$$
C_{*}(\lambda):=C C_{1} K\left(\frac{1}{\lambda-\varepsilon}+\frac{1}{\alpha-\lambda}\right) \quad \text { for } \lambda \in(\varepsilon, \alpha),
$$

and take $\delta \in\left(0, \delta_{1}\right]$ and $r>0$ sufficiently small so that

$$
\zeta_{*}(\delta) \max \left\{\max _{\lambda \in\left[\eta_{0}, \hat{\eta}\right]} C_{*}(\lambda), C C_{1} K\left(\frac{1}{\varepsilon}+\frac{1}{\alpha}\right)\right\}<\frac{1}{2}
$$

and

$$
r<\min \left(\delta, \frac{\delta}{2 C}\right)
$$

Let $\eta$ be a positive number in the interval $\left[\eta_{0}, \hat{\eta}\right]$, and $Y_{\eta}$ the Banach space $B C^{\eta}\left(\boldsymbol{R}^{-} ; \mathscr{B}\right)$. For $(\psi, y) \in B_{E^{c u}}(r) \times \bar{B}_{Y_{\eta}}(\delta)$ set $\mathscr{F}_{\delta}(\psi, y)(t):=V(t) \psi-$ $\mathscr{K}_{\delta}(y)(t), t \in \boldsymbol{R}^{-}$, where $\mathscr{K}_{\delta}(y)(t)$ is defined as follows:

$$
\mathscr{K}_{\delta}(y)(t):=\lim _{n \rightarrow \infty} \int_{t}^{0} V(t-s) \Pi^{c u} \Gamma^{n} f_{\delta}(y(s)) d s-\lim _{n \rightarrow \infty} \int_{-\infty}^{t} V(t-s) \Pi^{s} \Gamma^{n} f_{\delta}(y(s)) d s .
$$

Proposition 1. For each $\psi \in B_{E^{c u}}(r), \mathscr{F}_{\delta}(\psi, \cdot)$ defines a contraction mapping from $\bar{B}_{Y_{\eta}}(\delta)$ into itself, and therefore has a unique fixed point $\Lambda_{*}(\psi)$. Moreover, $\Lambda_{*}$ is a Lipschitz mapping from $B_{E^{c u}}(r)$ to $Y_{\eta}$ with Lipschitz constant $2 C$.

Proof. Let $y \in \bar{B}_{Y_{\eta}}(\delta)$. It follows from (18) that

$$
\begin{aligned}
\left\|\left[\Pi^{c u} \mathscr{F}_{\delta}(\psi, y)\right](t)\right\|_{\mathscr{B}} e^{\eta t} & \leq e^{\eta t}\left(C e^{-\varepsilon t}\|\psi\|_{\mathscr{B}}+\int_{t}^{0} C C_{1} K e^{-\varepsilon(t-s)} \delta \zeta_{*}(\delta) d s\right) \\
& \leq C r+C C_{1} K \delta \zeta_{*}(\delta) / \varepsilon
\end{aligned}
$$

and that

$$
\begin{aligned}
\left\|\left[\Pi^{s} \mathscr{F}_{\delta}(\psi, y)\right](t)\right\|_{\mathscr{B}} e^{\eta t} & \leq e^{\eta t} \int_{-\infty}^{t} C C_{1} K e^{-\alpha(t-s)} \delta \zeta_{*}(\delta) d s \\
& \leq\left(C C_{1} K \delta \zeta_{*}(\delta) / \alpha\right) e^{\eta t} .
\end{aligned}
$$


Hence we see from $(20)$ and $(21)$ that $\left\|\mathscr{F}_{\delta}(\psi, y)\right\|_{Y_{\eta}}<\delta$, and therefore $\mathscr{F}_{\delta}(\psi, y)$ is well-defined. Again by (18) it follows also that

$$
\begin{aligned}
\left\|\mathscr{F}_{\delta}\left(\psi, y_{1}\right)-\mathscr{F}_{\delta}\left(\psi, y_{2}\right)\right\|_{Y_{\eta}} \leq & \sup _{t \leq 0} e^{\eta t} \int_{t}^{0} C C_{1} K \zeta_{*}(\delta) e^{-\varepsilon(t-s)}\left\|y_{1}-y_{2}\right\|_{Y_{\eta}} e^{-\eta s} d s \\
& +\sup _{t \leq 0} e^{\eta t} \int_{-\infty}^{t} C C_{1} K \zeta_{*}(\delta) e^{-\alpha(t-s)}\left\|y_{1}-y_{2}\right\|_{Y_{\eta}} e^{-\eta s} d s \\
\leq & C_{*}(\eta) \zeta_{*}(\delta)\left\|y_{1}-y_{2}\right\|_{Y_{\eta}}
\end{aligned}
$$

for $y_{1}, y_{2} \in \bar{B}_{Y_{\eta}}(\delta)$, and hence by $(20)$ that $\mathscr{F}_{\delta}(\psi, \cdot)$ is a contraction form $\bar{B}_{Y_{\eta}}(\delta)$ into itself.

Let $\psi_{1}, \psi_{2} \in B_{E^{c u}}(r)$. Then (20) implies

$$
\begin{aligned}
\left\|\Lambda_{*}\left(\psi_{1}\right)-\Lambda_{*}\left(\psi_{2}\right)\right\|_{Y_{\eta}}= & \left\|\mathscr{F}_{\delta}\left(\psi_{1}, \Lambda_{*}\left(\psi_{1}\right)\right)-\mathscr{F}_{\delta}\left(\psi_{2}, \Lambda_{*}\left(\psi_{2}\right)\right)\right\|_{Y_{\eta}} \\
\leq & \left\|V^{c u}(\cdot)\right\|_{\mathscr{L}\left(E^{c u} ; Y_{\eta}\right)}\left\|\psi_{1}-\psi_{2}\right\|_{\mathscr{B}} \\
& +C_{*}(\eta) \zeta_{*}(\delta)\left\|\Lambda_{*}\left(\psi_{1}\right)-\Lambda_{*}\left(\psi_{2}\right)\right\|_{Y_{\eta}},
\end{aligned}
$$

from which, together with $\left\|V^{c u}(\cdot)\right\|_{\mathscr{L}\left(E^{c u} ; Y_{\eta}\right)} \leq C, V^{c u}(\cdot)$ being $\left.V(\cdot)\right|_{E^{c u}}$, the latter part of the proposition immediately follows.

We observe from (22) that $\left\|\Pi^{s} \Lambda_{*}(\psi)(t)\right\|_{\mathscr{B}}<\delta$ for $t \leq 0$. Notice also that $\Lambda_{*}(\psi)$ is independent of the choice of $\eta \in\left[\eta_{0}, \hat{\eta}\right]$ since $Y_{\eta_{1}} \subset Y_{\eta_{2}}$ for $\eta_{1}<\eta_{2}$ and $\mathscr{F}_{\delta}$ is a contraction. Let $H_{*}: E^{c u} \rightarrow E^{s}$ be the mapping defined by $H_{*}(\psi):=$ $\Pi^{s} \circ \mathrm{ev}_{0} \circ \Lambda_{*}(\psi)$ for $\psi \in B_{E^{c u}}(r)$ and set $W_{\mathrm{loc}}^{c u}(r, \delta):=\operatorname{graph} H_{*}$.

Proposition 2. $W_{\mathrm{loc}}^{c u}(r, \delta)$ is locally invariant for Eq. (7), i.e., the statements (a) and (b) of Theorem 7 (ii) are valid.

Proof. The proof is almost the same as that of Theorem 1 (iii). Let $\xi \in W_{\mathrm{loc}}^{c u}(r, \delta)$. Then there is a $\psi \in B_{E^{c u}}(r)$ with $\xi=\psi+H_{*}(\psi)$. Since $\Lambda_{*}(\psi)$ is the fixed point of $\mathscr{F}_{\delta}(\psi, \cdot)$, we have

$$
\begin{aligned}
\Lambda_{*}(\psi)(t)= & V(t) \psi-\lim _{n \rightarrow \infty} \int_{t}^{0} V(t-s) \Pi^{c u} \Gamma^{n} f_{\delta}\left(\Lambda_{*}(\psi)(s)\right) d s \\
& +\lim _{n \rightarrow \infty} \int_{-\infty}^{t} V(t-s) \Pi^{s} \Gamma^{n} f_{\delta}\left(\Lambda_{*}(\psi)(s)\right) d s
\end{aligned}
$$

for $t \in \boldsymbol{R}^{-}$. Since $\left\|\Lambda_{*}(\psi)\right\|_{Y_{\eta}}<\delta$ and $\xi=\Lambda_{*}(\psi)(0)$, Lemma 2 implies that $\Lambda_{*}(\psi)(t)=\xi_{t}=x_{t}\left(0, \xi ; f_{\delta}\right)$ for $t \in \boldsymbol{R}^{-}$. From (21) and the continuity of $x_{t}\left(0, \xi ; f_{\delta}\right)$ there exists a $t_{\xi}>0$ such that $\Pi^{c u} x_{t}\left(0, \xi ; f_{\delta}\right) \in B_{E^{c u}}(r)$ and $\sup _{|t| \leq t_{\xi}}\left(\left\|\Pi^{c u} x_{t}\left(0, \xi ; f_{\delta}\right)\right\|_{\mathscr{B}}+\left\|\Pi^{s} x_{t}\left(0, \xi ; f_{\delta}\right)\right\|_{\mathscr{B}}\right)<\delta$. Now let $\tau$ be given in such a way that $|\tau| \leq t_{\xi}$, and put $\chi:=\Pi^{c u} x_{\tau}\left(0, \xi ; f_{\delta}\right)$ and also $\tau_{*}:=\max \{0, \tau\}$. 
Notice that $v(t):=x\left(t+\tau, 0, \xi ; f_{\delta}\right)$ is a solution of Eq. (7) with $f_{\delta}$ in place of $f$ satisfying $v_{t}=\Lambda_{*}(\psi)(t+\tau)$ for $t \leq-\tau_{*}$, so that

$$
\sup _{t \leq 0}\left\|v_{t}\right\|_{\mathscr{B}} e^{\eta t} \leq \max \left(\left\|\Lambda_{*}(\psi)\right\|_{Y_{\eta}} e^{-\eta \tau_{*}}, \sup _{0 \leq s \leq \tau_{*}}\left\|x_{s}\left(0, \xi ; f_{\delta}\right)\right\|_{\mathscr{B}} e^{\eta\left(s-\tau_{*}\right)}\right)<\delta .
$$

Hence by Lemma 2 again, combined with Proposition 1, we have $v(t)=$ $\left[\Lambda_{*}(\chi)(t)\right](0)$ for $t \in \boldsymbol{R}^{-}$, and therefore

$$
x_{\tau}\left(0, \xi ; f_{\delta}\right)=v_{0}=\Lambda_{*}(\chi)(0)=\chi+H_{*}(\chi) \in W_{\mathrm{loc}}^{c u}(r, \delta) .
$$

Since both $\sup _{|t| \leq t_{\xi}}\left\|\Pi^{c u} x_{t}\left(0, \xi ; f_{\delta}\right)\right\|_{\mathscr{B}}$ and $\sup _{|t| \leq t_{\xi}}\left\|\Pi^{s} x_{t}\left(0, \xi ; f_{\delta}\right)\right\|_{\mathscr{B}}$ are less than $\delta$, we observe that $f_{\delta}\left(x_{t}\left(0, \xi ; f_{\delta}\right)\right)=f\left(x_{t}\left(0, \xi ; f_{\delta}\right)\right)$ for $|t| \leq t_{\xi}$. By virtue of Theorem 1 and the uniquness of solutions for the initial value problem, one can conclude that $x_{t}(0, \xi ; f)=x_{t}\left(0, \xi ; f_{\delta}\right)$ for $|t| \leq t_{\xi}$, from which, together with (25), $x_{\tau}(0, \xi ; f) \in W_{\mathrm{loc}}^{c u}(r, \delta)$ follows. So the property (a) holds. To certify (b) set

$$
\Omega:=\left\{\phi \in \mathscr{B}:\left\|\Pi^{c u} \phi\right\|_{\mathscr{B}}+\left\|\Pi^{s} \phi\right\|_{\mathscr{B}}<\delta,\left\|\Pi^{c u} \phi\right\|_{\mathscr{B}}<r\right\} .
$$

If $x_{t}\left(0, \xi ; f_{\delta}\right)$ belongs to $\Omega$ for any $t \in[0, T]$, consider $\chi$ in the above for $\tau \in[0, T]$. Then one can obtain the estimate (24) with $T$ in place of $\tau_{*}$, so that in the same way as above, (25) holds for $\tau \in[0, T]$. Consequently, (b) is also valid.

Proposition 3. The behavior of solutions of Eq. (7) on $W_{\mathrm{loc}}^{c u}(r, \delta)$ is completely determined by the ordinary differential equation (17) in the sense of Theorem 7 (iii).

Proof. Suppose that $x$ is a solution of Eq. (7) with $x_{t} \in W_{\mathrm{loc}}^{c u}(r, \delta)$ on $J$. Then it follows that $\Pi^{s} x_{t}=H_{*}(\Phi z(t))$ on $J$, and therefore $f\left(\Phi z(t)+\Pi^{s} x_{t}\right)=$ $f\left(\Phi z(t)+H_{*}(\Phi z(t))\right)$ on $J$. Since $z(t)$ is a solution of (16), this implies that $z(t)$ satisfies (17) for $t \in J$.

Conversely, let $z(t)$ be a solution of Eq. (17) on $J$ such that $\Phi z(t) \in \Omega$ for $t \in J, \Omega$ being the one given by (26). Then $z$ can be uniquely extended on $\boldsymbol{R}$ as a solution, denoted by $z(t)$ again, of

$$
\dot{z}=G z+\left\langle x^{*}, f_{\delta}\left(\Phi z+H_{*}(\Phi z)\right)\right\rangle
$$

because of $\left|\left\langle x^{*}, f_{\delta}\left(\Phi z+H_{*}(\Phi z)\right)\right\rangle\right| \leq K \delta \zeta_{*}(\delta)\left(\sum_{i=1}^{d}\left\|\psi_{i}\right\|_{\mathscr{B}^{*}}^{2}\right)^{1 / 2}$, where $|\cdot|$ stands for the Euclidean norm in $\boldsymbol{R}^{d}$. Let $\xi(t):=\Phi_{z}(t)+H_{*}\left(\Phi_{z}(t)\right)$. Then it follows from $[18$, Theorem 4.2] that $x(t):=[\xi(t)](0)$ is a solution of Eq. (3) with $h(t)=$ $f_{\delta}\left(\Phi z(t)+H_{*}(\Phi z(t))\right)$ and satisfies $x_{t}=\xi(t)$. Since

$$
f_{\delta}\left(\Phi z(t)+H_{*}(\Phi z(t))\right)=f\left(\Phi z(t)+H_{*}(\Phi z(t))\right)=f\left(x_{t}\right) \quad \text { for } t \in J,
$$

$x(t)$ is a solution of Eq. (7) on $J$ satisfying $\Pi^{c u} x_{t}=\Phi z(t)$ on $J$. 
Thus, it remains to be shown that the manifold $W_{\text {loc }}^{c u}(r, \delta)$ is $C^{k}$-smooth and is tangent to $E^{c u}$ at 0 . For this it suffices to prove $H_{*}$, and hence $\Lambda_{*}$, are of class $C^{k}$ as well as $D H_{*}(0)=0$ since it is given as the graph of $H_{*}=\Pi^{s} \circ \mathrm{ev}_{0} \circ$ $\Lambda_{*}$. In fact, we will deduce that $\Lambda_{*}(\psi)$ is $C^{k}$-smooth if we regard $\Lambda_{*}$ as a map taking values in the space $Y_{\hat{\eta}}$.

In what follows we denote by $Y_{\lambda}^{(p)}$ the Banach space $B C^{\lambda}\left(\boldsymbol{R}^{-} ; \mathscr{L}^{p}\left(E^{c u} ; \mathscr{B}\right)\right)$ for $\lambda \geq 0$ and $p=1,2, \ldots, k$. Also, by the same notation $J_{v}$ with $v \geq 0$ we mean the inclusion mappings from $B C^{\lambda}\left(\boldsymbol{R}^{-} ; U\right)$ to $B C^{\lambda+v}\left(\boldsymbol{R}^{-} ; U\right)$ for all $\lambda \geq 0$ and any Banach space $U$. Clearly, $J_{v} \in \mathscr{L}\left(B C^{\lambda}\left(\boldsymbol{R}^{-} ; U\right) ; B C^{\lambda+v}\left(\boldsymbol{R}^{-} ; U\right)\right)$.

Proposition 4. $\Lambda_{*}$ is of class $C^{k}$ as a mapping from $B_{E^{c u}}(r)$ to $Y_{\hat{\eta}}$.

Proof. The theorem can be proved by a similar argument to the proof of the smoothness of the center manifold for an equilibrium of some nonlinear semiflow [6, Theorem 7. 7; Chapter IX]. We first take positive numbers $\eta_{q} \in(\eta, \hat{\eta})$ so that $\eta_{q}>q \eta_{q-1}$ for $q=1, \ldots, k$. Let us recall that for any $\eta \in\left[\eta_{0}, \hat{\eta}\right], \Lambda_{*}(\psi)$ is the fixed point of the mapping $F_{\delta}(\psi, \cdot)$ in $Y_{\eta}$.

Step 1: Differentiability.

Differentiating (23) formally in $\psi$, we have

$$
\begin{aligned}
{\left[\left[D \Lambda_{*}(\psi)\right](t)\right] \phi=} & V(t) \phi-\lim _{n \rightarrow \infty} \int_{t}^{0} V(t-s) \Pi^{c u} \Gamma^{n}\left(D f_{\delta}\left(\Lambda_{*}(\psi)(s)\right)\left[\left[D \Lambda_{*}(\psi)\right](s)\right] \phi\right) d s \\
& +\lim _{n \rightarrow \infty} \int_{-\infty}^{t} V(t-s) \Pi^{s} \Gamma^{n}\left(D f_{\delta}\left(\Lambda_{*}(\psi)(s)\right)\left[\left[D \Lambda_{*}(\psi)\right](s)\right] \phi\right) d s
\end{aligned}
$$

for $\phi \in E^{c u}$ and $t \in \boldsymbol{R}^{-}$. Let us now justify this. Given $\mu \in[0, \hat{\eta}]$ and $v \in B C^{\mu}\left(\boldsymbol{R}^{-} ; \mathscr{L}(\mathscr{B} ; X)\right)$, we consider a mapping $\mathscr{H}_{0}(v): Y_{\lambda} \rightarrow Y_{\lambda+\mu}$ defined by

$$
\begin{aligned}
{\left[\mathscr{H}_{0}(v) w_{0}\right](t):=} & \lim _{n \rightarrow \infty} \int_{t}^{0} V(t-s) \Pi^{c u} \Gamma^{n} v(s) w_{0}(s) d s \\
& -\lim _{n \rightarrow \infty} \int_{-\infty}^{t} V(t-s) \Pi^{s} \Gamma^{n} v(s) w_{0}(s) d s
\end{aligned}
$$

for $w_{0} \in Y_{\lambda}$; here $\lambda \geq 0$ with $\lambda+\mu \leq \hat{\eta}$. For any $t \in \boldsymbol{R}^{-}$and $w_{0} \in Y_{\lambda}$ it follows that

$$
\begin{aligned}
\left\|\left[\mathscr{H}_{0}(v) w_{0}\right](t)\right\|_{\mathscr{B}} \leq & \int_{t}^{0} C C_{1} K \zeta_{*}(\delta) e^{-\varepsilon(t-s)}\left(\|v\|_{\mu, \mathscr{L}(\mathscr{B} ; X)} e^{-\mu s}\right)\left(\left\|w_{0}\right\|_{Y_{\lambda}} e^{-\lambda s}\right) d s \\
& +\int_{-\infty}^{t} C C_{1} K \zeta_{*}(\delta) e^{-\alpha(t-s)}\left(\|v\|_{\mu, \mathscr{L}(\mathscr{B} ; X)} e^{-\mu s}\right)\left(\left\|w_{0}\right\|_{Y_{\lambda}} e^{-\lambda s}\right) d s \\
\leq & C_{*}(\lambda+\mu)\|v\|_{\mu, \mathscr{L}(\mathscr{B} ; X)}\left\|w_{0}\right\|_{Y_{\lambda}} e^{-(\lambda+\mu) t},
\end{aligned}
$$


hence

$$
\left\|\mathscr{H}_{0}(v) w_{0}\right\|_{Y_{\lambda+\mu}} \leq C_{*}(\lambda+\mu)\|v\|_{\mu, \mathscr{L}(\mathscr{B} ; X)}\left\|w_{0}\right\|_{Y_{\lambda}}, \quad w_{0} \in Y_{\lambda} .
$$

$\mathscr{H}_{0}(v)$ also defines a bounded linear map, denoted $\mathscr{H}(v)$, from $Y_{\lambda}^{(1)}$ to $Y_{\lambda+\mu}^{(1)}$ by letting

$$
[[\mathscr{H}(v) w](t)] \phi:=\left[\mathscr{H}_{0}(v) w \phi\right](t) \quad \text { for } w \in Y_{\lambda}^{(1)} \text { and } \phi \in E^{c u},
$$

where $w \phi \in Y_{\lambda}$ is given by $(w \phi)(t):=w(t) \phi$ for $t \in \boldsymbol{R}^{-}$, and (27) yields the estimate

$$
\|\mathscr{H}(v) w\|_{Y_{\lambda+\mu}^{(1)}} \leq C_{*}(\lambda+\mu)\|v\|_{\mu, \mathscr{L}(\mathscr{B} ; X)}\|w\|_{Y_{\lambda}^{(1)}}, \quad w \in Y_{\lambda}^{(1)} .
$$

Because $V^{c u}(\cdot)$ belongs to $\mathscr{L}\left(E^{c u} ; Y_{\eta}\right)=Y_{\eta}^{(1)}$, we thus find that $D \Lambda_{*}(\psi)$ ought to be a solution of the linear equation, parametrized by $\psi \in B_{E^{c u}}(r)$, in the space $Y_{\eta}^{(1)}$ :

$$
A_{1}=V^{c u}(\cdot)-\mathscr{H}\left(\widetilde{D f_{\delta}}\left(\Lambda_{*}(\psi)\right)\right) A_{1} .
$$

It follows from (28) with $\lambda=\eta$ and $\mu=0, \quad$ (20) and the fact $\left\|\widetilde{D f_{\delta}}\left(\Lambda_{*}(\psi)\right)\right\|_{0, \mathscr{L}(\mathscr{B} ; X)} \leq \zeta_{*}(\delta)$ that $\left\|\mathscr{H}\left(\widetilde{D f_{\delta}}\left(\Lambda_{*}(\psi)\right)\right)\right\|_{\mathscr{L}\left(Y_{\eta}^{(1)}\right)} \leq 1 / 2$ holds, from which $A_{1}=A_{1}(\psi)$ is uniquely determined for each $\psi \in B_{E^{c u}}(r)$ and is given by

$$
\begin{aligned}
A_{1}(\psi) & =\left(I_{Y_{\eta}^{(1)}}+\mathscr{H}\left(\widetilde{D f_{\delta}}\left(\Lambda_{*}(\psi)\right)\right)\right)^{-1} V^{c u}(\cdot) \\
& =\sum_{n=0}^{\infty}(-1)^{n}\left(\mathscr{H}\left(\widetilde{D f_{\delta}}\left(\Lambda_{*}(\psi)\right)\right)\right)^{n} V^{c u}(\cdot) .
\end{aligned}
$$

Let us take any $v$ and $\eta$ such that $v \in\left[\eta_{1}, \hat{\eta}\right]$ and $\eta \in\left(\eta_{0}, \eta_{1}\right)$. We will verify that $J_{v-\eta} \Lambda_{*}(\psi)$ is differentiable and $D\left(J_{v-\eta} \Lambda_{*}(\psi)\right)=J_{v-\eta} A_{1}(\psi)$. Since (23) may be written as $\Lambda_{*}(\psi)=V^{c u}(\cdot) \psi-\mathscr{K}_{\delta}\left(\Lambda_{*}(\psi)\right)$, we see from (29) and (30) that

$$
\begin{aligned}
&-\left[\Lambda_{*}(\psi+h)-\Lambda_{*}(\psi)-A_{1}(\psi) h\right] \\
&=-V^{c u}(\cdot) h+\mathscr{K}_{\delta}\left(\Lambda_{*}(\psi+h)\right)-\mathscr{K}_{\delta}\left(\Lambda_{*}(\psi)\right)+A_{1}(\psi) h \\
&=-\left(A_{1}(\psi)+\mathscr{H}\left(\widetilde{D f}_{\delta}\left(\Lambda_{*}(\psi)\right)\right) A_{1}(\psi)\right) h+\mathscr{K}_{\delta}\left(\Lambda_{*}(\psi+h)\right) \\
&-\mathscr{K}_{\delta}\left(\Lambda_{*}(\psi)\right)+A_{1}(\psi) h \\
&= \mathscr{K}_{\delta}\left(\Lambda_{*}(\psi+h)\right)-\mathscr{K}_{\delta}\left(\Lambda_{*}(\psi)\right)-\mathscr{H}_{0}\left(\widetilde{D f}_{\delta}\left(\Lambda_{*}(\psi)\right)\right) A_{1}(\psi) h,
\end{aligned}
$$

for $h \in E^{c u}$ with $\|h\|_{\mathscr{B}}$ small. Noting that

$$
f_{\delta}\left(\Lambda_{*}(\psi+h)(s)\right)-f_{\delta}\left(\Lambda_{*}(\psi)(s)\right)=\int_{0}^{1} D f_{\delta}\left(\Lambda_{\sigma}(\psi)(s)\right) d \sigma\left(\Lambda_{*}(\psi+h)(s)-\Lambda_{*}(\psi)(s)\right),
$$


where $\Lambda_{\sigma}(\psi):=(1-\sigma) \Lambda_{*}(\psi)+\sigma \Lambda_{*}(\psi+h)$ for $\sigma \in[0,1]$, we have

$$
\mathscr{K}_{\delta}\left(\Lambda_{*}(\psi+h)\right)-\mathscr{K}_{\delta}\left(\Lambda_{*}(\psi)\right)=\mathscr{H}_{0}\left(L_{\psi, h}\right)\left(\Lambda_{*}(\psi+h)-\Lambda_{*}(\psi)\right),
$$

where $L_{\psi, h}$ is an element in $B C\left(\boldsymbol{R}^{-} ; \mathscr{L}(\mathscr{B} ; X)\right)$ defined by

$$
L_{\psi, h}(s):=\int_{0}^{1} D f_{\delta}\left(\Lambda_{\sigma}(\psi)(s)\right) d \sigma \quad \text { for } s \in \boldsymbol{R}^{-} .
$$

Hence

$$
\begin{aligned}
-\left[\Lambda_{*}(\psi+h)-\Lambda_{*}(\psi)-A_{1}(\psi) h\right] \\
=\mathscr{H}_{0}\left(L_{\psi, h}-\widetilde{D f_{\delta}}\left(\Lambda_{*}(\psi)\right)\right)\left(\Lambda_{*}(\psi+h)-\Lambda_{*}(\psi)\right) \\
\quad+\mathscr{H}_{0}\left(\widetilde{D f_{\delta}}\left(\Lambda_{*}(\psi)\right)\right)\left(\Lambda_{*}(\psi+h)-\Lambda_{*}(\psi)-A_{1}(\psi) h\right),
\end{aligned}
$$

and therefore by (27) and (20)

$$
\begin{aligned}
&\left\|J_{v-\eta} \Lambda_{*}(\psi+h)-J_{v-\eta} \Lambda_{*}(\psi)-J_{v-\eta} A_{1}(\psi) h\right\|_{Y_{v}} \\
& \leq C_{*}(v)\left\|L_{\psi, h}-\widetilde{D f_{\delta}}\left(\Lambda_{*}(\psi)\right)\right\|_{v-\eta, \mathscr{L}(\mathscr{B} ; X)}\left\|\Lambda_{*}(\psi+h)-\Lambda_{*}(\psi)\right\|_{Y_{\eta}} \\
&+C_{*}(v)\left\|\widetilde{D f_{\delta}}\left(\Lambda_{*}(\psi)\right)\right\|_{0, \mathscr{L}(\mathscr{B} ; X)}\left\|J_{v-\eta} \Lambda_{*}(\psi+h)-J_{v-\eta} \Lambda_{*}(\psi)-J_{v-\eta} A_{1}(\psi) h\right\|_{Y_{v}} \\
& \leq C_{*}(v)\left\|L_{\psi, h}-\widetilde{D f_{\delta}}\left(\Lambda_{*}(\psi)\right)\right\|_{v-\eta, \mathscr{L}(\mathscr{B} ; X)}\left\|\Lambda_{*}(\psi+h)-\Lambda_{*}(\psi)\right\|_{Y_{\eta}} \\
&+(1 / 2)\left\|J_{v-\eta} \Lambda_{*}(\psi+h)-J_{v-\eta} \Lambda_{*}(\psi)-J_{v-\eta} A_{1}(\psi) h\right\|_{Y_{v}} .
\end{aligned}
$$

Since

$$
L_{\psi, h}-\widetilde{D f_{\delta}}\left(\Lambda_{*}(\psi)\right)=\int_{0}^{1}\left(\widetilde{D f_{\delta}}\left(\Lambda_{\sigma}(\psi)\right)-\widetilde{D f_{\delta}}\left(\Lambda_{*}(\psi)\right)\right) d \sigma
$$

in $B C^{\nu-\eta}\left(\boldsymbol{R}^{-} ; \mathscr{L}(\mathscr{B} ; X)\right)$, Proposition 1, combined with (20), yields

$$
\begin{aligned}
& \left\|J_{v-\eta} \Lambda_{*}(\psi+h)-J_{v-\eta} \Lambda_{*}(\psi)-J_{v-\eta} A_{1}(\psi) h\right\|_{Y_{v}} \\
& \quad \leq 4 C C_{*}(v) \sup _{0 \leq \sigma \leq 1}\left\|\widetilde{D f_{\delta}}\left(\Lambda_{\sigma}(\psi)\right)-\widetilde{D f_{\delta}}\left(\Lambda_{*}(\psi)\right)\right\|_{v-\eta, \mathscr{L}(\mathscr{B} ; X)}\|h\|_{\mathscr{B}} .
\end{aligned}
$$

Observe that $\sup _{0 \leq \sigma \leq 1}\left\|\Lambda_{\sigma}(\psi)-\Lambda_{*}(\psi)\right\|_{Y_{\eta}} \rightarrow 0$ as $\|h\|_{\mathscr{B}} \rightarrow 0$, because of the inequality

$$
\begin{aligned}
\left\|\Lambda_{\sigma}(\psi)-\Lambda_{*}(\psi)\right\|_{Y_{\eta}} & =\left\|\sigma\left(\Lambda_{*}(\psi+h)-\Lambda_{*}(\psi)\right)\right\|_{Y_{\eta}} \\
& \leq\left\|\Lambda_{*}(\psi+h)-\Lambda_{*}(\psi)\right\|_{Y_{\eta}} \\
& \leq 2 C\|h\|_{\mathscr{B}} .
\end{aligned}
$$


Since $\sup _{\phi \in S_{\delta}}\left\|D f_{\delta}(\phi)\right\|_{\mathscr{L}(\mathscr{B} ; X)} \leq \zeta_{*}(\delta)$, the mapping $\widetilde{D f_{\delta}}: B C^{v}\left(\boldsymbol{R}^{-} ; S_{\delta}\right) \rightarrow$ $B C^{\nu-\eta}\left(\boldsymbol{R}^{-} ; \mathscr{L}(\mathscr{B} ; X)\right)$ is continuous by the fact stated in section 2.4. Hence we see that $\sup _{0 \leq \sigma \leq 1}\left\|\widetilde{D f_{\delta}}\left(\Lambda_{\sigma}(\psi)\right)-\widetilde{D f_{\delta}}\left(\Lambda_{*}(\psi)\right)\right\|_{v-\eta, \mathscr{L}(\mathscr{B} ; X)} \rightarrow 0$ as $\|h\|_{\mathscr{B}} \rightarrow 0$, which implies the differentiability of the mapping $J_{v-\eta} \Lambda_{*}(\psi)$ together with the relation $D\left(J_{v-\eta} \Lambda_{*}(\psi)\right)=J_{v-\eta} A_{1}(\psi)$.

Step 2: $C^{1}$-smoothness.

Let $v \in\left[\eta_{1}, \hat{\eta}\right]$. In this paragraph, we consider $\Lambda_{*}(\psi)$ as an element in $Y_{\eta_{1}}$, and prove the $C^{1}$-smoothness of the mapping $\Lambda_{*}$. By the fact established in Step 1, we know that $\Lambda_{*}(\psi)$ is differentiable and it satisfies $D \Lambda_{*}(\psi)=$ $J_{\eta_{1}-\eta} A_{1}(\psi)$, where $\eta \in\left(\eta_{0}, \eta_{1}\right)$ and $A_{1}(\psi)$ is an element in $Y_{\eta}^{(1)}$ which is given by (30). Therefore, in order to establish the $C^{1}$-smoothness of $\Lambda_{*}$ in $Y_{v}^{(1)}$, it suffices to prove the continuity of the mapping $J_{v-\eta} A_{1}$. Observe that

$$
J_{v-\eta} A_{1}(\psi)=\sum_{n=0}^{\infty}(-1)^{n} J_{v-\eta}\left(\mathscr{H}\left(\widetilde{D f_{\delta}}\left(\Lambda_{*}(\psi)\right)\right)\right)^{n} V^{c u}(\cdot) .
$$

This series in $Y_{v}^{(1)}$ converges uniformly for $\psi \in B_{E^{c u}}(r)$, because the norm of each term $J_{v-\eta}\left(\mathscr{H}\left(\widetilde{D f}_{\delta}\left(\Lambda_{*}(\psi)\right)\right)\right)^{n}$ does not exceed $(1 / 2)^{n}$ as seen in Step 1. We will certify the continuity of the term $J_{v-\eta}\left(\mathscr{H}\left(\widetilde{D f_{\delta}}\left(\Lambda_{*}(\psi)\right)\right)\right)^{n}$ as a mapping from $B_{E^{c u}}(r)$ to $\mathscr{L}\left(Y_{\eta}^{(1)}, Y_{v}^{(1)}\right)$. Given positive integer $n$ put $a_{1}:=\left(\eta_{1}-\eta\right) / n$. It is then easy to see that as a mapping from $Y_{\eta}^{(1)}$ to $Y_{v}^{(1)}$

$$
\begin{aligned}
J_{v-\eta}\left(\mathscr{H}\left(\widetilde{D f_{\delta}}\left(\Lambda_{*}(\psi)\right)\right)\right)^{n} & =J_{v-\eta_{1}} J_{a_{1}}^{n}\left(\mathscr{H}\left(\widetilde{D f_{\delta}}\left(\Lambda_{*}(\psi)\right)\right)\right)^{n} \\
& =J_{v-\eta_{1}}\left(J_{a_{1}} \mathscr{H}\left(\widetilde{D f_{\delta}}\left(\Lambda_{*}(\psi)\right)\right)\right)^{n}=J_{v-\eta_{1}}\left(\mathscr{H}\left(J_{a_{1}} \widetilde{D f_{\delta}}\left(\Lambda_{*}(\psi)\right)\right)\right)^{n}
\end{aligned}
$$

holds for $\psi \in B_{E^{c u}}(r)$. Since $\sup _{\phi \in S_{\delta}}\left\|D f_{\delta}(\phi)\right\|_{\mathscr{L}(\mathscr{B} ; X)} \leq \zeta_{*}(\delta)$ again, the mapping $J_{a_{1}} \widetilde{D f_{\delta}}: B C^{\eta_{1}}\left(\boldsymbol{R}^{-} ; S_{\delta}\right) \rightarrow B C^{a_{1}}\left(\boldsymbol{R}^{-} ; \mathscr{L}(\mathscr{B} ; X)\right)$ is continuous by the fact stated in section 2.4. Then $\mathscr{H}\left(J_{a_{1}} \widetilde{D f_{\delta}}\left(\Lambda_{*}(\psi)\right)\right)$ is continuous in $\psi$; and therefore so is $J_{v-\eta}\left(\mathscr{H}\left(\widetilde{D_{\delta}}\left(\Lambda_{*}(\psi)\right)\right)\right)^{n}$. Thus the continuity of $J_{v-\eta} A_{1}: B_{E^{c u}}(r) \rightarrow Y_{v}^{(1)}$ follows.

Consequently, the proof of the proposition in case of $k=1$ is completed. Indeed, we may put $v=\hat{\eta}$ in Step 2. Next we will treat the case $k=2$ or $k \geq 3$ in the subsequent Steps.

Step 3: $C^{2}$-smoothness.

Let $v \in\left[\eta_{2}, \hat{\eta}\right]$. We consider $\Lambda_{*}(\psi)$ as an element in $Y_{\eta_{1}}$, and prove the $C^{2}$-smoothness of the mapping $J_{v-\eta_{1}} \Lambda_{*}$. Take a constant $\eta \in\left(\eta_{0}, \eta_{1}\right)$. By virtue of the fact shown in Step 1 and Step 2, it follows that $D\left(J_{v-\eta_{1}} \Lambda_{*}(\psi)\right)=$ $J_{v-\eta_{1}} D \Lambda_{*}(\psi)=J_{v-\eta_{1}} J_{\eta_{1}-\eta} A_{1}(\psi)=J_{v-\eta_{2}} J_{\eta_{2}-\eta} A_{1}(\psi)$, where

$$
J_{\eta_{2}-\eta} A_{1}(\psi)=\sum_{n=0}^{\infty}(-1)^{n} J_{\eta_{2}-\eta}\left(\mathscr{H}\left(\widetilde{D f_{\delta}}\left(\Lambda_{*}(\psi)\right)\right)\right)^{n} V^{c u}(\cdot) .
$$


Thus, in order to estabilish the $C^{2}$-smoothness of $\Lambda_{*}$ it suffices to prove the $C^{1}$-smoothness of the mapping $J_{\eta_{2}-\eta} A_{1}$, and hence the uniform convergence of the series in $Y_{\eta_{2}}^{(2)}$ formally given by

$$
\begin{aligned}
\sum_{n=0}^{\infty}( & -1)^{n} D\left(J_{\eta_{2}-\eta}\left(\mathscr{H}\left(\widetilde{D f_{\delta}}\left(\Lambda_{*}(\psi)\right)\right)\right)^{n}\right) V^{c u}(\cdot) \\
& =\sum_{n=0}^{\infty}(-1)^{n} D\left(J_{\eta_{2}-\eta_{1}}\left(\mathscr{H}\left(\widetilde{D f_{\delta}}\left(\Lambda_{*}(\psi)\right)\right)\right)^{n}\right) J_{\eta_{1}-\eta} V^{c u}(\cdot),
\end{aligned}
$$

together with the continuity of each term $D\left(J_{\eta_{2}-\eta_{1}}\left(\mathscr{H}\left(\widetilde{D_{\delta}}\left(\Lambda_{*}(\psi)\right)\right)\right)^{n}\right) J_{\eta_{1}-\eta} V^{c u}(\cdot)$ in $\psi$. Since $\eta_{2}>2 \eta_{1}$, we can choose an $\eta^{\prime} \in\left(\eta_{1}, \eta_{2}-\eta_{1}\right)$. Note that $\sup _{\phi \in S_{\delta}}\left\|D^{2} f_{\delta}(\phi)\right\|_{\mathscr{L}(\mathscr{B} ; \mathscr{L}(\mathscr{B} ; X))} \leq M_{1}$ and $\eta^{\prime}>\eta_{1}$. Then the mapping $J_{\eta^{\prime}} \widetilde{D f_{\delta}}$ : $B C^{\eta_{1}}\left(\boldsymbol{R}^{-} ; S_{\delta}\right) \rightarrow B C^{\eta^{\prime}}\left(\boldsymbol{R}^{-} ; \mathscr{L}(\mathscr{B} ; X)\right)$ is of class $C^{1}$ by the fact stated in section 2.4. Hence $J_{\eta^{\prime}} \widetilde{D f_{\delta}} \circ \Lambda_{*}: E^{c u} \rightarrow B C^{\eta^{\prime}}\left(\boldsymbol{R}^{-} ; \mathscr{L}(\mathscr{B} ; X)\right)$ is of class $C^{1}$ and

$$
\begin{aligned}
D\left(J_{\eta^{\prime}} \mathscr{H}\left(\widetilde{D f_{\delta}}\left(\Lambda_{*}(\psi)\right)\right)\right) & =\mathscr{H} D\left(J_{\eta^{\prime}} \widetilde{D f_{\delta}}\left(\Lambda_{*}(\psi)\right)\right) \\
& =\mathscr{H} J_{\eta^{\prime}} \widetilde{D^{2} f_{\delta}}\left(\Lambda_{*}(\psi)\right) D \Lambda_{*}(\psi) \\
& =\mathscr{H} J_{\eta^{\prime}} \widetilde{D^{2} f_{\delta}}\left(\Lambda_{*}(\psi)\right)\left(J_{\eta_{1}-\eta} A_{1}(\psi)\right)
\end{aligned}
$$

in $\mathscr{L}\left(E^{c u} ; \mathscr{L}\left(Y_{\eta_{1}}^{(1)} ; Y_{\eta_{1}+\eta^{\prime}}^{(1)}\right)\right)$. Now given an integer $n>1$ let $a_{2}>0$ be the positive number satisfying $\eta^{\prime}+(n-1) a_{2}=\eta_{2}-\eta_{1}$. Since $J_{\eta_{2}-\eta_{1}}=J_{a_{2}}^{n-1} J_{\eta^{\prime}}$, it follows from (31) that

$$
\begin{aligned}
& D\left(J_{\eta_{2}-\eta_{1}}\left(\mathscr{H}\left(\widetilde{D f_{\delta}}\left(\Lambda_{*}(\psi)\right)\right)\right)^{n}\right) \\
&=\sum_{i=1}^{n}\left(\mathscr{H}\left(J_{a_{2}} \widetilde{D f_{\delta}}\left(\Lambda_{*}(\psi)\right)\right)\right)^{i-1} \cdot\left(\mathscr{H} J_{\eta^{\prime}} \widetilde{D^{2} f_{\delta}}\left(\Lambda_{*}(\psi)\right)\left(J_{\eta_{1}-\eta} A_{1}(\psi)\right)\right) \\
& \cdot\left(\mathscr{H}\left(J_{a_{2}} \widetilde{D f_{\delta}}\left(\Lambda_{*}(\psi)\right)\right)\right)^{n-i} \quad \text { in } \mathscr{L}\left(E^{c u} ; \mathscr{L}\left(Y_{\eta_{1}}^{(1)} ; Y_{\eta_{2}}^{(1)}\right)\right) .
\end{aligned}
$$

Note that the factors $\mathscr{H}\left(J_{a_{2}} \widetilde{D f_{\delta}}\left(\Lambda_{*}(\psi)\right)\right)$ and $\mathscr{H} J_{\eta^{\prime}} \widetilde{D^{2} f_{\delta}}\left(\Lambda_{*}(\psi)\right)\left(J_{\eta_{1}-\eta} A_{1}(\psi)\right)$ are all continuous in $\psi \in B_{E^{c u}}(r)$. Furthermore, from (28), (20) and the fact $\left\|\widetilde{D f_{\delta}}\left(\Lambda_{*}(\psi)\right)\right\|_{a_{2}, \mathscr{L}(\mathscr{B} ; X)} \leq\left\|\widetilde{D f_{\delta}}\left(\Lambda_{*}(\psi)\right)\right\|_{0, \mathscr{L}(\mathscr{B} ; X)} \leq \zeta_{*}(\delta)$ it follows that for $\lambda \in\left[\eta_{1}, \eta_{2}-a_{2}\right]$

$$
\begin{aligned}
\left\|\mathscr{H}\left(J_{a_{2}} \widetilde{D f_{\delta}}\left(\Lambda_{*}(\psi)\right)\right)\right\|_{\mathscr{L}\left(Y_{\lambda}^{(1)} ; Y_{\lambda+a_{2}}^{(1)}\right)} & \leq C_{*}\left(\lambda+a_{2}\right)\left\|\widetilde{D f_{\delta}}\left(\Lambda_{*}(\psi)\right)\right\|_{a_{2}, \mathscr{L}(\mathscr{B} ; X)} \\
& \leq\left(2 \zeta_{*}(\delta)\right)^{-1} \cdot \zeta_{*}(\delta)=1 / 2 .
\end{aligned}
$$

Notice also that $\mathscr{L}\left(E^{c u} ; B C^{\eta^{\prime}}\left(\boldsymbol{R}^{-} ; \mathscr{L}(\mathscr{B} ; X)\right)\right)=B C^{\eta^{\prime}}\left(\boldsymbol{R}^{-} ; \mathscr{L}\left(E^{c u} ; \mathscr{L}(\mathscr{B} ; X)\right)\right)$, and from (28) that $\|\mathscr{H}\|_{\mathscr{L}\left(B C \eta^{\prime} ; \mathscr{L}\left(Y_{\lambda}^{(1)} ; Y_{\lambda+\eta^{\prime}}^{(1)}\right)\right.} \leq C_{*}\left(\lambda+\eta^{\prime}\right)$ holds. It then follows from (19) and (20) that 


$$
\begin{aligned}
\| \mathscr{H} J_{\eta^{\prime}} & \widetilde{D^{2}} f_{\delta}\left(\Lambda_{*}(\psi)\right)\left(J_{\eta_{1}-\eta} A_{1}(\psi)\right) \|_{\mathscr{L}\left(E^{c u} ; \mathscr{L}\left(Y_{\lambda}^{(1)} ; Y_{\lambda+\eta^{\prime}}^{(1)}\right)\right)} \\
& \leq\|\mathscr{H}\|_{\mathscr{L}\left(B C^{\eta^{\prime}} ; \mathscr{L}\left(Y_{\lambda}^{(1)} ; Y_{\lambda+\eta^{\prime}}^{(1)}\right)\right)}\left\|J_{\eta^{\prime}} \widetilde{D^{2} f_{\delta}}\left(\Lambda_{*}(\psi)\right)\left(J_{\eta_{1}-\eta} A_{1}(\psi)\right)\right\|_{\mathscr{L}\left(E^{c u} ; B C^{\prime}\right)} \\
& \leq C_{*}\left(\lambda+\eta^{\prime}\right)\left\|J_{\eta^{\prime}} \widetilde{D^{2} f_{\delta}}\left(\Lambda_{*}(\psi)\right)\left(J_{\eta_{1}-\eta} A_{1}(\psi)\right)\right\|_{\mathscr{L}\left(E^{c u} ; B C^{\prime}\right)} \\
& \leq C_{*}\left(\lambda+\eta^{\prime}\right)\left\|\widetilde{D^{2} f_{\delta}}\left(\Lambda_{*}(\psi)\right)\right\|_{\eta^{\prime}-\eta_{1}, \mathscr{L}(\mathscr{B} ; \mathscr{L}(\mathscr{B} ; X))}\left\|J_{\eta_{1}-\eta} A_{1}(\psi)\right\|_{\eta_{\eta_{1}}} \\
& \leq\left(2 \zeta_{*}(\delta)\right)^{-1} M_{1}\left\|A_{1}(\psi)\right\|_{Y_{\eta}^{(1)}}, \quad \lambda \in\left[\eta_{1}, \eta_{2}-\eta^{\prime}\right],
\end{aligned}
$$

where $B C^{\eta^{\prime}}$ stands for $B C^{\eta^{\prime}}\left(\boldsymbol{R}^{-} ; \mathscr{L}(\mathscr{B} ; X)\right)$. Hence by (32), (33) and (34)

$$
\begin{aligned}
& \left\|D\left(J_{\eta_{2}-\eta_{1}}\left(\mathscr{H}\left(\widetilde{D f_{\delta}}\left(\Lambda_{*}(\psi)\right)\right)\right)^{n}\right) J_{\eta_{1}-\eta} V^{c u}(\cdot)\right\|_{Y_{\eta_{2}}^{(2)}} \\
& \quad \leq \sum_{i=1}^{n}(1 / 2)^{i-1} \cdot\left(2 \zeta_{*}(\delta)\right)^{-1} M_{1}\left\|A_{1}(\psi)\right\|_{Y_{\eta}^{(1)}} \cdot(1 / 2)^{n-i} \cdot\left\|V^{c u}(\cdot)\right\|_{Y_{\eta}^{(1)}} \\
& \quad \leq n 2^{-n+1} \zeta_{*}(\delta)^{-1} M_{1} C^{2} \quad \text { for } \psi \in B_{E^{c u}}(r),
\end{aligned}
$$

where we used the facts that $V^{c u}(\cdot) \in \mathscr{L}\left(E^{c u} ; Y_{\eta}\right)=Y_{\eta}^{(1)}$ with $\left\|V^{c u}(\cdot)\right\|_{Y_{\eta}^{(1)}} \leq C$ and that, by (30), $\sup _{\psi \in B_{E} \text { cu }(r)}\left\|A_{1}(\psi)\right\|_{Y_{\eta}^{(1)}} \leq 2 C$ holds. Consequently, the uniform convergence of the series $\sum_{n=0}^{\infty} D\left(J_{\eta_{2}-\eta_{1}}\left(\mathscr{H}\left(\widetilde{D f_{\delta}}\left(\Lambda_{*}(\psi)\right)\right)\right)^{n}\right) J_{\eta_{1}-\eta} V^{c u}(\cdot)$ in $Y_{\eta_{2}}^{(2)}$ is guaranteed, as required.

Step 4: $C^{k}$-smoothness.

Even for the verification of $C^{k}$-smoothness of $\Lambda_{*}$, our approach employed in Step 3 to show the $C^{2}$-smoothness is available. In the following, however, to avoid several cumbersome notation and confusion, we will sketch the proof for the restricted case $k=3$, and omit the proof of the general case.

Let $v \in\left[\eta_{3}, \hat{\eta}\right]$. We consider $\Lambda_{*}(\psi)$ as an element in $Y_{\eta_{2}}$, and prove the $C^{3}$-smoothness of the mapping $J_{v-\eta_{2}} \Lambda_{*}$. Take a constant $\eta \in\left(\eta_{0}, \eta_{1}\right)$. Then $D\left(J_{v-\eta_{2}} \Lambda_{*}(\psi)\right)=J_{v-\eta_{3}} J_{\eta_{3}-\eta} A_{1}(\psi)$ and

$$
J_{\eta_{3}-\eta} A_{1}(\psi)=\sum_{n=0}^{\infty}(-1)^{n} J_{\eta_{3}-\eta}\left(\mathscr{H}\left(\widetilde{D f_{\delta}}\left(\Lambda_{*}(\psi)\right)\right)\right)^{n} V^{c u}(\cdot) .
$$

Thus, in order to estabilish the $C^{3}$-smoothness of $\Lambda_{*}$ it suffices to certify the uniform convergence of the (formal) series in $Y_{\eta_{3}}^{(3)}$

$$
\begin{aligned}
& \sum_{n=0}^{\infty}(-1)^{n} D^{2}\left(J_{\eta_{3}-\eta}\left(\mathscr{H}\left(\widetilde{D f_{\delta}}\left(\Lambda_{*}(\psi)\right)\right)\right)^{n}\right) V^{c u}(\cdot) \\
& \quad=\sum_{n=0}^{\infty}(-1)^{n} D^{2}\left(J_{\eta_{3}-\eta_{2}}\left(\mathscr{H}\left(\widetilde{D f_{\delta}}\left(\Lambda_{*}(\psi)\right)\right)\right)^{n}\right) J_{\eta_{2}-\eta} V^{c u}(\cdot),
\end{aligned}
$$


together with the continuity of each term $D\left(J_{\eta_{3}-\eta_{2}}\left(\mathscr{H}\left(\widetilde{D f_{\delta}}\left(\Lambda_{*}(\psi)\right)\right)\right)^{n}\right) J_{\eta_{2}-\eta} V^{c u}(\cdot)$ in $\psi$. Since $\eta_{3}>3 \eta_{2}$, we can choose an $\eta^{\prime \prime} \in\left(2 \eta_{2}, \eta_{3}-\eta_{2}\right)$. Note that the supremum of the norm of $D^{i} f_{\delta}(\phi)$ over $S_{\delta}$ does not exceed $M_{1}$ for $i=1,2,3$. Therefore, since $2 \eta_{2}<\eta^{\prime \prime}$, by the fact stated in section 2.4 the mapping $J_{\eta^{\prime \prime}} \widetilde{D f_{\delta}}: B C^{\eta_{2}}\left(\boldsymbol{R}^{-} ; S_{\delta}\right) \rightarrow B C^{\eta^{\prime \prime}}\left(\boldsymbol{R}^{-} ; \mathscr{L}(\mathscr{B} ; X)\right)$ is of class $C^{2}$, and moreover $J_{\eta^{\prime \prime} / 2} \widetilde{D f_{\delta}}: B C^{\eta_{2}}\left(\boldsymbol{R}^{-} ; S_{\delta}\right) \rightarrow B C^{\eta^{\prime \prime} / 2}\left(\boldsymbol{R}^{-} ; \mathscr{L}(\mathscr{B} ; X)\right) \quad$ is of class $C^{1}$. Hence $J_{\eta^{\prime \prime}} \widetilde{D f_{\delta}} \circ \Lambda_{*}: E^{c u} \rightarrow B C^{\eta^{\prime \prime}}\left(\boldsymbol{R}^{-} ; \mathscr{L}(\mathscr{B} ; X)\right)$ is of class $C^{2}$ and

$$
\begin{aligned}
D^{2}\left(J_{\eta^{\prime \prime}} \mathscr{H}\left(\widetilde{D f_{\delta}}\left(\Lambda_{*}(\psi)\right)\right)\right)= & \mathscr{H} D^{2}\left(J_{\eta^{\prime \prime}} \widetilde{D f_{\delta}}\left(\Lambda_{*}(\psi)\right)\right) \\
= & \mathscr{H} D\left(J_{\eta^{\prime \prime}} \widetilde{D^{2} f_{\delta}}\left(\Lambda_{*}(\psi)\right)\left(D \Lambda_{*}(\psi)\right)\right) \\
= & \mathscr{H} J_{\eta^{\prime \prime}} \widetilde{D^{3} f_{\delta}}\left(\Lambda_{*}(\psi)\right)\left(D \Lambda_{*}(\psi), D \Lambda_{*}(\psi)\right) \\
& +\mathscr{H} J_{\eta^{\prime \prime}} \widetilde{D^{2} f_{\delta}}\left(\Lambda_{*}(\psi)\right)\left(D^{2} \Lambda_{*}(\psi)\right) .
\end{aligned}
$$

Now, given an integer $n>1$, put $a_{3}:=\left(\eta_{3}-\eta_{2}-\eta^{\prime \prime}\right) /(n-1)$. Then $a_{3}>0$ satisfies

$$
\eta_{3}-\eta_{2}=(n-1) a_{3}+\eta^{\prime \prime}=(n-2) a_{3}+\left(\eta^{\prime \prime} / 2+\eta^{\prime \prime} / 2\right)+a_{3} .
$$

Hence, corresponding to (32) we get the expansion

$$
D^{2}\left(J_{\eta_{3}-\eta_{2}}\left(\mathscr{H}\left(\widetilde{D f_{\delta}}\left(\Lambda_{*}(\psi)\right)\right)\right)^{n}\right)=\Sigma_{1}+\Sigma_{2}+\Sigma_{3},
$$

where

$$
\begin{aligned}
\Sigma_{1}= & \sum_{i=1}^{n}\left(\mathscr{H}\left(J_{a_{3}} \widetilde{D f_{\delta}}\left(\Lambda_{*}(\psi)\right)\right)\right)^{i-1} \cdot\left(\mathscr{H} J_{\eta^{\prime \prime}} \widetilde{D^{3} f_{\delta}}\left(\Lambda_{*}(\psi)\right)\left(D \Lambda_{*}(\psi), D \Lambda_{*}(\psi)\right)\right) \\
& \cdot\left(\mathscr{H}\left(J_{a_{3}} \widetilde{D f_{\delta}}\left(\Lambda_{*}(\psi)\right)\right)\right)^{n-i}, \\
\Sigma_{2}= & \sum_{i=1}^{n}\left(\mathscr{H}\left(J_{a_{3}} \widetilde{D f_{\delta}}\left(\Lambda_{*}(\psi)\right)\right)\right)^{i-1} \cdot\left(\mathscr{H} J_{\eta^{\prime \prime}} \widetilde{D^{2} f_{\delta}}\left(\Lambda_{*}(\psi)\right)\left(D^{2} \Lambda_{*}(\psi)\right)\right) \\
& \cdot\left(\mathscr{H}\left(J_{a_{3}} \widetilde{D f_{\delta}}\left(\Lambda_{*}(\psi)\right)\right)\right)^{n-i}, \\
\Sigma_{3}= & \sum_{i, j=1 ; i+j \leq n}^{n-1} J_{a_{3}}\left(\mathscr{H}\left(J_{a_{3}} \widetilde{D f_{\delta}}\left(\Lambda_{*}(\psi)\right)\right)\right)^{i-1} \cdot\left(\mathscr{H} J_{\eta^{\prime \prime} / 2} \widetilde{D^{2} f_{\delta}}\left(\Lambda_{*}(\psi)\right)\left(D \Lambda_{*}(\psi)\right)\right) \\
& \cdot\left(\mathscr{H}\left(J_{a_{3}} \widetilde{D f_{\delta}}\left(\Lambda_{*}(\psi)\right)\right)\right)^{n-i-j} \cdot\left(\mathscr{H} J_{\eta^{\prime \prime} / 2} \widetilde{D^{2} f_{\delta}}\left(\Lambda_{*}(\psi)\right)\left(D \Lambda_{*}(\psi)\right)\right) \\
& \cdot\left(\mathscr{H}\left(J_{a_{3}} \widetilde{D f_{\delta}}\left(\Lambda_{*}(\psi)\right)\right)\right)^{j-1} .
\end{aligned}
$$

Note that $\eta^{\prime \prime} / 2>\eta_{2}$. Therefore each term in $\Sigma_{1}, \Sigma_{2}$ and $\Sigma_{3}$ is continuous in $\psi \in B_{E^{c u}}(r)$. Furthermore, through the calculation similar to (33) and (34) we 
get an estimate on the norm of each term in $\Sigma_{1}, \Sigma_{2}$ and $\Sigma_{3}$; indeed, each norms of their terms do not exceed $K_{1} \times(1 / 2)^{n}$, where $K_{1}$ is some constant which does not depend on $n$. Therefore, the sum of all the norms of terms in $\Sigma_{1}, \Sigma_{2}$ and $\Sigma_{3}$ does not exceed $K_{2} \times n^{2} / 2^{n}$ for some constant $K_{2}$ which does not depend on $n$; consequently, the series $\sum_{n=0}^{\infty}(-1)^{n} D^{2}\left(J_{\eta_{3}-\eta_{2}}\left(\mathscr{H}\left(\widetilde{D f}_{\delta}\left(\Lambda_{*}(\psi)\right)\right)\right)^{n}\right) J_{\eta_{2}-\eta} V^{c u}(\cdot)$ is convergent in $Y_{\eta_{3}}^{(3)}$ uniformly for $\psi \in B_{E^{c u}}(r)$, as required.

Finally, in view of (29) an argument similar to (13) in the proof of Theorem 5 yields Property (i) of Theorem 7. The proof of Theorem 7 is now completed.

\section{Center manifolds and center-stable manifolds for FDE}

We can also establish the existence of smooth local center manifolds and center-stable manifolds for Eq. (7) by almost the same arguments as in the previous section.

We first note that the following lemma can be established analogously to Lemma 1; we omit the proof.

Lemma 3. Let $\psi \in E^{c}$, and $\eta$ be a constant less than $\alpha$. If $x$ is a solution of Eq. (7) with the properties that $\Pi^{c} x_{0}=\psi, \sup _{t \in \boldsymbol{R}}\left\|x_{t}\right\|_{\mathscr{B}} e^{-\eta|t|}<\infty$ and $\sup _{t \in \boldsymbol{R}}\left\|f\left(x_{t}\right)\right\|_{X}<\infty$, then

$$
\begin{aligned}
x_{t}= & V(t) \psi+\lim _{n \rightarrow \infty} \int_{0}^{t} V(t-s) \Pi^{c} \Gamma^{n} f\left(x_{s}\right) d s-\lim _{n \rightarrow \infty} \int_{t}^{\infty} V(t-s) \Pi^{u} \Gamma^{n} f\left(x_{s}\right) d s \\
& +\lim _{n \rightarrow \infty} \int_{-\infty}^{t} V(t-s) \Pi^{s} \Gamma^{n} f\left(x_{s}\right) d s, \quad t \in \boldsymbol{R} .
\end{aligned}
$$

Conversely, if $y \in C(\boldsymbol{R} ; \mathscr{B})$ with $\sup _{t \in \boldsymbol{R}}\|y(t)\|_{\mathscr{B}} e^{-\eta|t|}<\infty$ and $\sup _{t \in \boldsymbol{R}}\|f(y(t))\|_{X}$ $<\infty$ satisfies

$$
\begin{aligned}
y(t)= & V(t) \psi+\lim _{n \rightarrow \infty} \int_{0}^{t} V(t-s) \Pi^{c} \Gamma^{n} f(y(s)) d \tau-\lim _{n \rightarrow \infty} \int_{t}^{\infty} V(t-s) \Pi^{u} \Gamma^{n} f(y(s)) d s \\
& +\lim _{n \rightarrow \infty} \int_{-\infty}^{t} V(t-s) \Pi^{s} \Gamma^{n} f(y(s)) d s, \quad t \in \boldsymbol{R},
\end{aligned}
$$

then the function $\xi(t)$ defined by

$$
\xi(t):=[y(t)](0), \quad t \in \boldsymbol{R}
$$

is a solution of (7) satisfying $\Pi^{c} \xi_{0}=\psi$, $\sup _{t \in \boldsymbol{R}}\left\|\xi_{t}\right\|_{\mathscr{B}} e^{-\eta|t|}<\infty$ and $\xi_{t}=y(t)$ for $t \in \boldsymbol{R}$.

Now let $\hat{\eta}, \eta_{0}$ and $\delta_{1}$ be the ones in the previous section, and choose $\delta \in\left(0, \delta_{1}\right]$ and $r>0$ sufficiently small such that, in place of $(20)$, 


$$
\zeta_{*}(\delta) \max \left\{\max _{\lambda \in\left[\eta_{0}, \hat{\eta}\right]} \hat{C}_{*}(\lambda), C C_{1} K\left(\frac{1}{\varepsilon}+\frac{2}{\alpha}\right)\right\}<\frac{1}{2}
$$

and (21) are satisfied, where

$$
\hat{C}_{*}(\lambda):=C C_{1} K\left(\frac{1}{\lambda-\varepsilon}+\frac{1}{\alpha+\lambda}+\frac{2}{\alpha-\lambda}\right) \quad \text { for } \lambda \in(\varepsilon, \alpha) .
$$

Fix $\eta \in\left[\eta_{0}, \hat{\eta} / k !\right]$, let $\hat{Y}_{\eta}$ be the Banach space $B C_{-\eta}(\boldsymbol{R} ; \mathscr{B})$ given by

$$
B C_{-\eta}(\boldsymbol{R} ; \mathscr{B}):=\left\{y \in C(\boldsymbol{R} ; \mathscr{B}): \sup _{t \in \boldsymbol{R}}\|y(t)\|_{\mathscr{B}} e^{-\eta|t|}<\infty\right\} .
$$

Here we notice that $\widetilde{D f}: B C_{-\eta}(\boldsymbol{R} ; \mathscr{B}) \rightarrow B C_{-\eta^{\prime}}(\boldsymbol{R} ; \mathscr{L}(\mathscr{B} ; X))$ is of class $C^{p}$ if $\eta^{\prime}>p \eta, p=1, \ldots, k$, by the same reasoning as the situations for Banach spaces $B C^{\lambda}\left(\boldsymbol{R}^{-} ; \mathscr{B}\right), \lambda \geq 0$ (see section 2.4 and [6]). In view of Lemma 3, let us set

$$
\begin{aligned}
\hat{\mathscr{F}_{\delta}}(\psi, y)(t):= & V(t) \psi+\lim _{n \rightarrow \infty} \int_{0}^{t} V(t-s) \Pi^{c} \Gamma^{n} f_{\delta}(y(s)) d s \\
& -\lim _{n \rightarrow \infty} \int_{t}^{\infty} V(t-s) \Pi^{u} \Gamma^{n} f_{\delta}(y(s)) d s \\
& +\lim _{n \rightarrow \infty} \int_{-\infty}^{t} V(t-s) \Pi^{s} \Gamma^{n} f_{\delta}(y(s)) d s
\end{aligned}
$$

for $(\psi, y) \in B_{E^{c}}(r) \times \bar{B}_{\hat{Y}_{\eta}}(\delta)$. Then, by a similar argument to the proof of Proposition 1 , one can see that $\hat{\mathscr{F}}_{\delta}(\psi, \cdot)$ defines a contraction map from $\bar{B}_{\hat{Y}_{\eta}}(\delta)$ into itself, and hence that it has a unique fixed point $\hat{\Lambda}_{*}(\psi)$ for each $\psi \in B_{E^{c}}(r)$. Notice that corresponding to $(22), \hat{\mathscr{F}}_{\delta}$ satisfies

$$
\begin{aligned}
\left\|\left[\Pi^{s} \hat{\mathscr{F}}_{\delta}(\psi, y)\right](t)\right\|_{\mathscr{B}} e^{-\eta|t|} & \leq e^{-\eta|t|} \int_{-\infty}^{t} C C_{1} K e^{-\alpha(t-s)} \delta \zeta_{*}(\delta) d s \\
& \leq\left(C C_{1} K \delta \zeta_{*}(\delta) / \alpha\right) e^{-\eta|t|}<\delta e^{-\eta|t|}
\end{aligned}
$$

so that $\left\|\Pi^{s} \hat{\Lambda}_{*}(\psi)(t)\right\|_{\mathscr{B}}<\delta$ for $t \in \boldsymbol{R}$. We can therefore establish the $C^{k}$ smoothness of $\hat{\Lambda}_{*}(\psi)$ besides the corresponding result to Proposition 2, that is, the local invariance of the graph of the mapping $\Pi^{s u} \circ \operatorname{ev}_{0} \circ \hat{\Lambda}_{*}$; the details will be omitted. Thus, for the existence and smoothness of the local center manifolds of the zero solution of Eq. (7) we have:

Theorem 8. Assume that $f \in C^{k}(\mathscr{B} ; X)$ with $f(0)=D f(0)=0$. Then there exist positive numbers $r, \delta$, and a $C^{k}$-mapping $\hat{H}_{*}: B_{E^{c}}(r) \rightarrow B_{E^{s u}}(\delta)$ with $\hat{H}_{*}(0)=0$, together with an open neighborhood $\Omega$ of 0 in $\mathscr{B}$, such that the following properties hold: 
(i) $W_{\text {loc }}^{c}(r, \delta):=\operatorname{graph} \hat{H}_{*}$ is tangent to $E^{c}$ at zero,

(ii) $W_{\mathrm{loc}}^{c}(r, \delta)$ is locally invariant for Eq. (7), that is,

(a) for any $\xi \in W_{\text {loc }}^{c}(r, \delta)$ there exists a $t_{\xi}>0$ such that $x_{t}(0, \xi ; f) \in$ $W_{\mathrm{loc}}^{c}(r, \delta)$ for $|t| \leq t_{\xi}$,

(b) if $\xi \in W_{\mathrm{loc}}^{c}(r, \delta)$ and $x_{t}(0, \xi ; f) \in \Omega$ for $0 \leq t \leq T$, then $x_{t}(0, \xi ; f) \in$ $W_{\mathrm{loc}}^{c}(r, \delta)$ for $0 \leq t \leq T$.

Similarly we can obtain the existence of the local center-stable manifold of the the zero solution of Eq. (7). Let $\hat{\eta}, \eta_{0}$ and $\eta$ be as above, and choose $\delta \in\left(0, \delta_{1}\right]$ and $r>0$ so small that $(20)$ and

$$
r<\min \left(\delta, \frac{\delta}{2 C}, \frac{\delta}{2 C C_{1}}\right)
$$

are satisfied. Let $\check{Y}_{\eta}$ be the Banach space $B C^{-\eta}\left(\boldsymbol{R}^{+} ; \mathscr{B}\right)$. Then the map $\check{\mathscr{F}}_{\delta}(\psi, \cdot)$ from $\bar{B}_{\check{Y}_{\eta}}(\delta)$ into itself, defined by

$$
\begin{aligned}
\check{\mathscr{F}_{\delta}}(\psi, y)(t):= & V(t) \psi+\lim _{n \rightarrow \infty} \int_{0}^{t} V(t-s) \Pi^{c s} \Gamma^{n} f_{\delta}(y(s)) d s \\
& -\lim _{n \rightarrow \infty} \int_{t}^{\infty} V(t-s) \Pi^{u} \Gamma^{n} f_{\delta}(y(s)) d s, \quad(\psi, y) \in B_{E^{c s}}(r) \times \bar{B}_{\check{Y}_{\eta}}(\delta),
\end{aligned}
$$

has a unique fixed point $\check{\Lambda}_{*}(\psi)$ for each $\psi \in B_{E^{c s}}(r)$. Corresponding to (22), $\check{\mathscr{F}}_{\delta}$ satisfies

$$
\begin{aligned}
\left\|\left[\Pi^{s} \check{\mathscr{F}}_{\delta}(\psi, y)\right](t)\right\|_{\mathscr{B}} e^{-\eta t} & \leq e^{-\eta t}\left(C C_{1} e^{-\alpha t}\|\psi\|_{\mathscr{B}}+\int_{0}^{t} C C_{1} K e^{-\alpha(t-s)} \delta \zeta_{*}(\delta) d s\right) \\
& \leq\left(C C_{1} r+C C_{1} K \delta \zeta_{*}(\delta) / \alpha\right) e^{-\eta t}<\delta e^{-\eta t}
\end{aligned}
$$

and in particular $\left\|\Pi^{s} \check{\Lambda}_{*}(\psi)(t)\right\|_{\mathscr{B}}<\delta$ for $t \geq 0$. The $C^{k}$-smoothness of $\check{\Lambda}_{*}(\psi)$ then follows from a similar argument to Proposition 4 , and hence the local existence and smoothness of center-stable manifolds of the zero solution of Eq. (7) are established. In summary we have:

Theorem 9. Assume that $f \in C^{k}(\mathscr{B} ; X)$ with $f(0)=D f(0)=0$. Then there exist positive numbers $r, \delta$, and a $C^{k}$-mapping $\check{H}_{*}: B_{E^{c s}}(r) \rightarrow B_{E^{u}}(\delta)$ with $\check{H}_{*}(0)=0$, together with an open neighborhood $\Omega$ of 0 in $\mathscr{B}$, such that the following properties hold:

(i) $W_{\mathrm{loc}}^{c s}(r, \delta):=\operatorname{graph} \check{H}_{*}$ is tangent to $E^{c s}$ at zero,

(ii) $W_{\mathrm{loc}}^{c s}(r, \delta)$ is locally invariant for Eq. (7), that is,

(a) for any $\xi \in W_{\mathrm{loc}}^{c s}(r, \delta)$ there exists a $t_{\xi}>0$ such that $x_{t}(0, \xi ; f) \in$ $W_{\mathrm{loc}}^{c s}(r, \delta)$ for $|t| \leq t_{\xi}$,

(b) if $\xi \in W_{\text {loc }}^{c s}(r, \delta)$ and $x_{t}(0, \xi ; f) \in \Omega$ for $0 \leq t \leq T$, then $x_{t}(0, \xi ; f) \in$ $W_{\mathrm{loc}}^{c s}(r, \delta)$ for $0 \leq t \leq T$. 


\section{Invariant manifolds for VDE}

In this section, we shall consider the Volterra difference equation in a Banach space $X$

$$
x(n+1)=\sum_{j=-\infty}^{n} Q(n-j) x(j)+g\left(x_{n}\right)
$$

as a discrete analogue of Eq. (7), where $\{Q(n)\}_{n>0}$ is a sequence of compact linear operators on $X$ and $g$ is a $C^{k}$-mapping from $\mathscr{B}^{\gamma}$ to $X$ with $g(0)=$ $D g(0)=0$.

Our objective here is to give generalizations of the results in [24] to an infinite-dimensional setting. We first show the existence of local smooth stable manifolds for Eq. (36) under the condition that the zero solution is hyperbolic. For $r>0$ and $\delta>0$ we set

$$
W_{\text {loc }}^{s}(r, \delta):=\left\{\phi \in \mathscr{B}^{\gamma}:\left\|\Pi^{s} \phi\right\|_{\mathscr{B}^{\gamma}}<r,\left\|x_{n}(0, \phi ; g)\right\|_{\mathscr{B}^{\gamma}}<\delta, n \in \boldsymbol{Z}^{+}\right\} .
$$

Then we get:

Theorem 10. Assume that the zero solution of (6) is hyperbolic and that $g \in C^{k}(\mathscr{B} ; X)$ with $g(0)=D g(0)=0$. Then there exist an $r>0$ and a $\delta>0$ such that $W_{\mathrm{loc}}^{s}(r, \delta)$ is a $C^{k}$-manifold diffeomorphic to an open set in $E^{s}$ with the properties

(i) $W_{\mathrm{loc}}^{s}(r, \delta)$ is tangent to $E^{s}$ at zero,

(ii) there are positive constants $M$ and $\beta$ such that

$$
\left\|x_{n}(0, \phi ; g)\right\|_{\mathscr{B}^{\gamma}} \leq M e^{-\beta n}\|\phi\|_{\mathscr{B}^{\gamma}}, \quad n \in \boldsymbol{Z}^{+}, \phi \in W_{\mathrm{loc}}^{s}(r, \delta),
$$

(iii) $W_{\mathrm{loc}}^{s}(r, \delta)$ is locally positively invariant for (36), that is, if $\phi \in W_{\mathrm{loc}}^{s}(r, \delta)$, we have $x_{v}(0, \phi ; g) \in W_{\mathrm{loc}}^{s}(r, \delta)$ for $v \in \boldsymbol{Z}^{+}$whenever $\Pi^{s} x_{v}(0, \phi ; g) \in$ $B_{E^{s}}(r)$.

The proof is analogous to that of Theorem 5: Let $\beta$ be a positive number less than $\alpha$, the one in Theorem 3 , and $\hat{Y}$ the Banach space $B^{\beta}\left(\boldsymbol{Z}^{+} ; \mathscr{B}^{\gamma}\right)$ (see section 2.4). One can obtain a similar result to Lemma 1 which characterizes $\boldsymbol{Z}^{+}$-bounded solutions of (36), i.e., solutions $x(n)$ satisfying $\sup _{n \in \boldsymbol{Z}^{+}}\left\|x_{n}\right\|_{\mathscr{B}^{\prime}}<\infty$. For $(\psi, y) \in B_{E^{c u}}\left(r_{0}\right) \times B_{\hat{Y}}(\delta)$ consider the map $\mathscr{G}$ defined by

$$
\begin{aligned}
\mathscr{G}(\psi, y)(n):= & y(n)-V(n) \psi+\sum_{j=0}^{n-1} V(n-j) \Pi^{s} \mathscr{E}(g(y(j))) \\
& -\sum_{j=n}^{\infty} V(n-j) \Pi^{u} \mathscr{E}(g(y(j)))
\end{aligned}
$$


for $n \in \boldsymbol{Z}^{+}$. Then, there exist appropriate positive numbers $r$ and $\delta$ such that $\mathscr{G}$ defines a $C^{k}$-mapping between $B_{E^{c u}}(r) \times B_{\hat{Y}}(\delta)$ and $\hat{Y}$, and $W_{\text {loc }}^{s}(r, \delta)$ can be characterized as the graph of the map $\Pi^{u} \circ \mathrm{ev}_{0} \circ \bar{\Lambda}_{*}: B_{E^{s}}(r) \rightarrow E^{u}$, where $\mathrm{ev}_{0}$ is the evaluation map and $\overline{\Lambda_{*}}(\psi)$ is the zero of $\mathscr{G}(\psi, \cdot)$, which is assured to be $C^{k}$-smooth in $\psi$ by the implicit function theorem. The rest of the proof is quite the same as that of Theorem 5 .

Similarly, for the existence of local unstable manifolds of the zero solution of Eq. (36) the following theorem holds.

Theorem 11. Assume that the zero solution of (6) is hyperbolic and that $g \in C^{k}\left(\mathscr{B}^{\gamma} ; X\right)$ with $g(0)=D g(0)=0$. Then there exist an $r>0$ and a $\delta>0$ such that $W_{\mathrm{loc}}^{u}(r, \delta)$ is a $C^{k}$-manifold diffeomorphic to an open set in $E^{u}$ with the properties

(i) $W_{\text {loc }}^{u}(r, \delta)$ is tangent to $E^{u}$ at zero,

(ii) there are positive constants $M$ and $\beta$ such that

$$
\left\|x_{n}(0, \phi ; g)\right\|_{\mathscr{B}^{\gamma}} \leq M e^{\beta n}\|\phi\|_{\mathscr{B}^{\gamma}}, \quad n \in \boldsymbol{Z}^{-}, \phi \in W_{\mathrm{loc}}^{u}(r, \delta)
$$

(iii) $W_{\mathrm{loc}}^{u}(r, \delta)$ is locally negatively invariant for (36), that is, if $\phi \in W_{\mathrm{loc}}^{u}(r, \delta)$, we have $x_{v}(0, \phi ; g) \in W_{\mathrm{loc}}^{u}(r, \delta)$ for $v \in \boldsymbol{Z}^{-}$whenever $\Pi^{u} x_{v}(0, \phi ; g) \in B_{E^{u}}(r)$.

Next we consider the case where the zero solution of (6) is not necessarily hyperbolic. Let $\bar{\Phi}$ and $\bar{\Psi}$ be the analogues in our setting of $\Phi$ and $\Psi$ in section 4 , respectively. It is easy to see that there is a $d \times d$-matrix $B$ with $\sigma(B)=\Sigma$ such that

$$
V^{c u}(n) \bar{\Phi}=\bar{\Phi} B^{n}, \quad n \in \boldsymbol{Z}^{+} .
$$

Then the $E^{c u}$-component of solutions of Eq. (36) can be related to those of some first order difference equation in $\boldsymbol{R}^{d}, d=\operatorname{dim} E^{c u}$; namely, if $x(n)$ is a solution of Eq. (36), then the function $z(n)$, given by $\bar{\Phi} z(n):=\Pi^{c u} x_{n}$, is a solution to the difference equation

$$
z(n+1)=B z(n)+\left\langle\bar{\Psi}, f\left(\bar{\Phi} z(n)+\Pi^{s} z_{n}\right)\right\rangle .
$$

On the existence of local center-unstable manifolds of the zero solution of Eq. (36) we have the following theorem.

Theorem 12. Assume that $g \in C^{k}\left(\mathscr{B}^{\gamma} ; X\right)$ with $g(0)=D g(0)=0$. Then there exist positive numbers $r, \delta$, and a $C^{k}$-mapping $h_{*}: B_{E^{c u}}(r) \rightarrow B_{E^{s}}(\delta)$ with $h_{*}(0)=0$, together with an open neighborhood $\Omega$ of 0 in $\mathscr{B}^{\gamma}$, such that the following properties hold:

(i) $W_{\operatorname{loc}}^{c u}(r, \delta):=\operatorname{graph} h_{*}$ is tangent to $E^{c u}$ at zero,

(ii) $W_{\mathrm{loc}}^{c u}(r, \delta)$ is locally invariant for Eq. (36), that is, 
(a) for any $\xi \in W_{\mathrm{loc}}^{c u}(r, \delta)$ there exists a positve integer $n_{\xi}$ such that $x_{n}(0, \xi ; g) \in W_{\text {loc }}^{\text {cu }}(r, \delta)$ for $|n| \leq n_{\xi}$,

(b) there exists an open neighborhood $\Omega$ of 0 in $\mathscr{B}^{\gamma}$ such that if $\xi \in W_{\mathrm{loc}}^{c u}(r, \delta)$ and $x_{n}(0, \xi ; g) \in \Omega$ for $0 \leq t \leq m$, then $x_{n}(0, \xi ; g) \in$ $W_{\mathrm{loc}}^{c u}(r, \delta)$ for $0 \leq n \leq m$.

(iii) If $x$ is a solution of Eq. (36) on a discrete interval $J$ such that $x_{n} \in W_{\mathrm{loc}}^{c u}(r, \delta)(n \in J)$, then the function $z(n)$ given by $\bar{\Phi} z(n):=\Pi^{c u} x_{n}$ satisfies the difference equation

$$
z(n+1)=B z(n)+\left\langle\bar{\Psi}, f\left(\bar{\Phi} z(n)+h_{*}(\bar{\Phi} z(n))\right)\right\rangle, \quad n \in J .
$$

Conversely, if $z(n)$ satisfy the equation (38) on an interval $J$ with $\bar{\Phi} z(n) \in \Omega(n \in J)$, then there exists a solution $x$ of Eq. (36) on $J$ such that $x_{n} \in W_{\mathrm{loc}}^{c u}(r, \delta)$ and $\Pi^{c u} x_{n}=\bar{\Phi} z(n)$ on $J$.

Moreover, correspondingly to Theorems 8 and 9, the local existence and smoothness of center manifolds and center-stable manifolds can be shown; we omit the statements and proofs of these theorems.

\section{Linearized stabilities and instabilities}

Applying some of the theorems in the preceeding sections, one can obtain criteria on stabilities and instabilities of the zero solution of Eq. (7) and Eq. (36), which may be viewed as the principle of linearized stability for functional differential equations and Volterra difference equations, respectively. See [29] for the results on functional differential equations. Here we will give the results for Volterra difference equations (see also [24]).

Theorem 13. Let $g \in C^{1}\left(\mathscr{B}^{\gamma}, X\right)$ satisfy $g(0)=D g(0)=0$, and suppose that the characteristic operator $z I_{X}-\sum_{n=0}^{\infty} Q(n) z^{-n}$ is invertible in $\mathscr{L}(X)$ for $|z| \geq 1$. Then the zero solution of Eq. (36) is exponentially asymptotically stable.

Proof. Since $\Sigma^{c u}=\varnothing$, it follows from Theorem 4 that $E^{c u}=\{0\}$ and therefore $W_{\text {loc }}^{s}(r, \delta)=B_{\mathscr{B} \gamma}(r)$. Moreover, by Theorem 10 (ii), we get

$$
\left\|x_{n}(\sigma, \phi ; g)\right\|_{\mathscr{B}^{\gamma}}=\left\|x_{n-\sigma}(0, \phi ; g)\right\|_{\mathscr{B}^{\gamma}} \leq M e^{-\beta(n-\sigma)}\|\phi\|_{\mathscr{B}^{\gamma}}, \quad n \geq \sigma, \phi \in B_{\mathscr{B}^{\gamma}}(r) .
$$

This completes the proof.

Theorem 14. Let $g \in C^{1}\left(\mathscr{B}^{\gamma}, X\right)$ satisfy $g(0)=D g(0)=0$, and suppose that the operator $z I_{X}-\sum_{n=0}^{\infty} Q(n) z^{-n}$ is not invertible in $\mathscr{L}(X)$ for some $|z|>1$. Then the zero solution of Eq. (36) is unstable.

Proof. By Theorem 12, there exists the local center-unstable manifold $W_{\text {loc }}^{c u}(r, \delta)$ of Eq. (36). By $g(0)=D g(0)=0$ we see that $\left\|g\left(\bar{\Phi} z+h_{*}(\bar{\Phi} z)\right)\right\|_{X}=$ 
$o(|z|)$. Also since $\sigma(B)=\Sigma^{c u}$ contains a number outside the unit disk in $C$, it follows from [7, Theorem 4. 40] that the zero solution of (37) is unstable. Thus, by Theorem 12 (iii) we conclude that the zero solution of (36) is unstable.

\section{References}

[1] Agarwal, R. P., Difference Equations and Inequalities, 2nd ed., Revised and Expanded: Theory, Methods and Applications, Marcel Dekker, New York, 2000.

[2] Aulbach, B. and Nguyen, V. M., Nonlinear semigroups and the existence, stability of semilinear nonautonomous evolution equations, Abstract and Applied Analysis, 1 (1996), 351-380.

[ 3 ] Carr, J. L., Applications of Center Manifold Theory, Springer-Verlag, New York, 1981.

[4] Cuevas, C. and Pinto, M., Asymptotic properties of solutions to nonaoutonomous Volterra difference systems with infinite delay, Advances in difference equations III, Comput. Math. Appl., 42 (2001), 551-559.

[5] Cuevas, C. and Pinto, M., Convergent solutions of linear functional difference equations in phase space, J. Math. Anal. Appl., 277 (2003), 324-341.

[6] Diekmann, O., van Gils, S. A., Verduyn Lunel, S. M., and Walther, H.-O., Delay Equations, Springer-Verlag, Berlin, New York, 1995.

[7] Elaydi, S., An Introduction to Difference Equations, 2nd ed., Springer-Verlag, New York, 1999.

[8] Elaydi S. and Murakami, S., Asymptotic stability versus exponential stability in linear Volterra difference equations of convolution type, J. Difference Equations and Appl., 2 (1996), 401-410.

[9] Elaydi, S. and Murakami, S., Uniform asymptotic stability in linear Volterra difference equations, J. Difference Equations and Appl., 3 (1998), 203-218.

[10] Furumochi, T., Murakami, S., and Nagabuchi, Y., Volterra difference equations on a Banach space and abstract differential equations with piecewise continuous delays, Japanese J. Math., 30 (2004), 387-412.

[11] Furumochi, T., Murakami, S., and Nagabuchi, Y., A generalization of Wiener's lemma and its application to Volterra difference equations on a Banach space, J. Difference Equations and Appl., 10 (2004), 1201-1214.

[12] Furumochi, T., Murakami, S., and Nagabuchi, Y., Stabilities in Volterra difference equations on a Banach space, Fields Institute Communications, 42 (2004), 159-175.

[13] Hale, J. K., Ordinary Differential Equations, John Wiley and Sons, New York, 1969.

[14] Hale, J. K. and Kato, J., Phase space for retarded equations with infinite delay, Funkcial. Ekvac., 21 (1978), 11-41.

[15] Hale, J. K. and Verduyn Lunel, S. M., Introduction to Functional Differential Equations, Springer-Verlag, Berlin, New York, 1993.

[16] Henry, D., Geometric Theory of Semilinear Parabolic Equations, Lecture Notes in Math., 804, Springer-Verlag, Berlin, New York, 1981.

[17] Hino, Y., Murakami, S., and Naito, T., Functional Differential Equations with Infinite Delay, Lecture Notes in Math., 1473, Springer-Verlag, Berlin, New York, 1991.

[18] Hino, Y., Murakami, S., Naito, Y., and Nguyen, V. M., A variation of constants formula for abstract functional differential equations in Banach spaces, J. Differential Equations, 179 (2002), 336-355. 
[19] Hino, Y., Murakami, S., and Nguyen, V. M., Decomposition of variation of constants formula for abstract functional differential equations, Funkcial. Ekvac., 45 (2002), 341-372.

[20] Hirsh, M. W., Pugh, C., and Shub, M., Invariant Manifolds, Lecture Notes in Math., 583, Springer-Verlag, Berlin, New York, 1977.

[21] Krisztin, T., Invariance and noninvariance of center manifolds of time- $t$ maps with respect to the semiflow, SIAM J. Math. Anal., 36 (2004/05), 717-739 (electronic).

[22] Krisztin, T., Walther, H.-O., and Wu, J., Shape, Smoothness, and Invariant Stratification of an Attracting Set for Delayed Monotone Positive Feedback, Field Institute Monographs, 11, Amer. Math. Soc., Providence, RI, 1999.

[23] Lang, S., Fundamentals of Differential Geometry, GTM 191, Springer-Verlag, New York, Berlin, Heidelberg, 1999.

[24] Matsunaga, H. and Murakami, S., Some invariatnt manifolds for functional difference equations with infinite delay, J. Difference Equations and Appl., 10 (2004), 661-689.

[25] Matsunaga, H. and Murakami, S., Asymptotic behavior of solutions of functional difference equations, J. Math. Anal. Appl., 305 (2005), 391-410.

[26] Memory, M. C., Stable and unstable manifolds for partial functional differential equations, Nonlinear Anal. TMA, 16 (1991), 131-142.

[27] Nguyen, V. M. and Wu, J., Invariant manifolds of partial functional differential equations, J. Differential Equations, 198 (2004), 381-421.

[28] Murakami, S., Naito, T., and Nguyen, V. M., Massera's theorem for almost periodic solutions of functional differential equations, J. Math. Soc. Japan, 56 (2004), 247-268.

[29] Murakami, S. and Nguyen, V. M., Some invariatnt manifolds for abstract functional differential equations and linearized stabilities, Vietnam J. Math., 30 (2002), 437-458.

[30] Nagabuchi, Y., Decomposition of phase space for linear Volterra difference equations in a Banach space, Funkcial. Ekvac., 49 (2006), 269-290.

[31] Shub, M., Global Stability of Dynamical Systems, Springer-Verlag, Berlin, New York, 1987.

[32] Wu, J., Theory and Applications of Partial Functional Differential Equations, Applied Math. Sci., 119, Springer-Verlag, Berlin, New York, 1996.

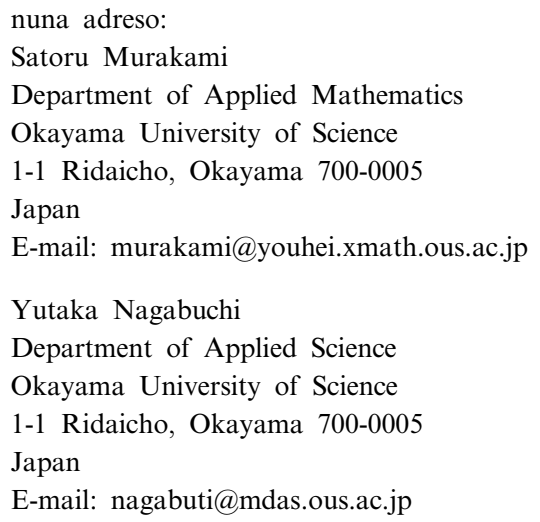

(Ricevita la 10-an de julio, 2006)

(Reviziita la 3-an de novembro, 2006) 